\title{
REPRESENTATIONS OF INTEGERS BY TERNARY QUADRATIC FORMS
}

\author{
BEN KANE
}

\begin{abstract}
We investigate the representation of integers by quadratic forms whose theta series lie in Kohnen's plus space $M_{3 / 2}^{+}(4 p)$, where $p$ is a prime. Conditional upon certain GRH hypotheses, we show effectively that every sufficiently large discriminant with bounded divisibility by $p$ is represented by the form, up to local conditions. We give an algorithm for explicitly calculating the bounds. For small $p$ we then use a computer to find the full list of all discriminants not represented by the form. Finally, conditional upon GRH for $L$-functions of weight 2 newforms, we give an algorithm for computing the implied constant of the Ramanujan-Petersson conjecture for weight $3 / 2$ cusp forms of level $4 N$ in Kohnen's plus space with $N$ odd and squarefree.
\end{abstract}

\section{INTRODUCTION}

Let $Q$ be a positive definite integral quadratic form in $m$ variables and let

$$
\theta_{Q}(\tau):=\sum_{x \in \mathbb{Z}^{m}} q^{Q(x)}
$$

be the associated theta series, where $q=e^{2 \pi i \tau}$. We will omit the subscript $Q$ when it is clear. Throughout this paper, a theta series will always mean $\theta_{Q}$ for some (mostly ternary) positive definite integral quadratic form $Q$. It is well known that $\theta$ is a modular form of weight $\frac{m}{2}$. For general information about quadratic forms, a good source is [19].

The natural question of which positive integers $n$ are represented by the form $Q$, that is whether there exists $x \in \mathbb{Z}^{m}$ such that $Q(x)=n$, has been studied extensively since Gauss.

One such well known result of Lagrange shows that every positive integer can be represented as the sum of four squares. The amazing " 15 theorem", proven first but unpublished by Conway and Schneeberger and recently shown by a much simpler method by Bhargava, asserts that a positive definite integral quadratic form represents every positive integer if and only if it represents the integers 1,2,3,5,6,7,10,14, and 15 [1]. Bhargava and Hanke have since shown that every integer valued quadratic form is universal if and only if it represents every integer less than 290 [2].

Date: June 24, 2008.

2000 Mathematics Subject Classification. 11E20, 11E45, $11 \mathrm{G} 05$.

Key words and phrases. Quaternion Algebra, Elliptic Curves, Maximal Orders, Half Integer Weight Modular Forms, Kohnen's Plus Space, Shimura Lifts. 
An eligible integer for a positive definite quadratic form $Q$ is a positive integer $n$ for which $Q(x)=n$ always has a local solution. We will call $D_{Q}\left(\right.$ resp. $\left.D_{\theta_{Q}}\right)$ a good bound for $Q\left(\right.$ resp. $\left.\theta_{Q}\right)$ if $Q$ represents every eligible integer greater than $D_{Q}\left(\operatorname{resp} . a_{\theta}(D)>0\right)$ where we have used $a_{f}(n)$ to denote the $n$-th Fourier coefficient of $f$.

Relying on the fact that $\theta$ is a modular form, Tartakowsky [29] effectively showed that every sufficiently large eligible integer is represented by $Q$ when $m \geq 5$. The corresponding result for $m=4$ was shown by the bounds of Kloosterman (the celebrated result of Deligne proved the optimal bound [5]).

We will study here the case $m=3$. Up to a technical complication at anisotropic primes and Schulze-Pillot's classification of finitely many spinor exceptional classes [26], bounds by Iwaniec [13] and Duke [7] for coefficients of half-integral weight cusp forms have shown that every sufficiently large eligible integer is represented.

Theorem (Duke- Schulze-Pillot [9]). If $Q$ is a positive definite quadratic form in 3 variables, then every sufficiently large eligible integer represented primitively by the Spinor Genus with bounded divisibility at the anisotropic primes is represented by $Q$.

This result is ineffective because it relies on a lower bound for the class numbers, while the best known effective result, due to Oesterlé [22], is insufficient for our purposes. Assuming GRH for Dirichlet $L$-functions, the result becomes effective, and hence under this assumption an algorithm must exist to determine which integers are represented.

By using a deep connection of Waldspurger [30] between half integer weight cusp forms and special values of $L$-series of weight 2 newforms, under the additional assumption of GRH for weight 2 modular forms, Ono and Soundararajan obtain a feasible bound of $2 \times 10^{10}$ for Ramanujan's ternary quadratic form $x^{2}+y^{2}+10 z^{2}$. With the help of a computer, they were able to prove the following.

Theorem (Ono-Soundararajan [24]). Conditional upon GRH, the eligible integers which are not represented by $x^{2}+y^{2}+10 z^{2}$ are exactly

$$
3,7,21,31,33,43,67,79,87,133,217,219,223,253,307,391,679,2719 .
$$

In this paper, we generalize the results of Ono and Soundararajan to develop an explicit algorithm for ternary quadratic forms $Q$ such that $\theta_{Q} \in M_{3 / 2}^{+}(4 p)$, the space of modular forms of weight $3 / 2$ and level $4 p$ in Kohnen's plus space (Ramanujan's form does not satisfy this condition). By the theory of modular forms, $\theta$ decomposes into

$$
\theta=E+\sum_{i=1}^{m} b_{i} g_{i}
$$

where $E$ is an Eisenstein series, $b_{i} \in \mathbb{C}$ and $g_{i}$ are fixed Hecke eigenforms in $S_{3 / 2}^{+}(4 p)$. We have already seen that the assumption of GRH for Dirichlet $L$-functions gives a bound of $D^{1 / 2-\epsilon}$ for $a_{E}(D)$. The fact that $\theta \in M_{3 / 2}^{+}(4 p)$ allows us to determine the coefficient explicitly. It then remains to bound the coefficients of $g_{i}$. 
Theorem 1.1. Let $N$ be squarefree and odd, $\delta>0$, and $g \in S_{3 / 2}^{+}(4 N)$ in the orthogonal complement of the space of lifts of one dimensional theta-series. Then, assuming GRH for L-functions of weight 2 newforms, there is an explicitly computable function $c_{\delta}$ such that

$$
\left|a_{g}(n)\right| \leq c_{\delta}\|g\| c_{N} \operatorname{dim}\left(S_{2}\left(\Gamma_{0}(N)\right)\right)^{\frac{1}{2}} N^{\frac{\delta}{2}} n^{\frac{1}{4}+\delta},
$$

where $a_{g}(n)$ denotes the $n$-th Fourier coefficient of $g,\|g\|$ denotes the Petersson norm of $g$, and $c_{N}$ is an explicitly computable function which grows like $N^{\epsilon}$.

Remark 1.2. Taking $\delta=\epsilon$, we obtain an explicit algorithm to determine the implied constant for the Ramanujan-Petersson conjecture in the $n$ aspect under GRH. However, $c_{\delta}$ is too large for practical purposes when $\delta$ is small.

We can now basically combine Theorem 1.1 with Littlewood's bound [20] for the Eisenstein series to obtain our desired (explicit) algorithm.

Remark 1.3. In practice, we will not bound $a_{E}(D)$ and $a_{g_{i}}(D)$ separately, but will rather bound the ratio, to obtain a better bound for our algorithm.

Theorem 1.4. Let $\theta \in M_{3 / 2}^{+}(4 p)$ be given. Assume GRH for Dirichlet L-functions and $L$-functions of weight 2 newforms. Then there exists an explicit algorithm which determines all fundamental discriminants $-D$ so that $D$ is not represented by $\theta$. Moreover, this algorithm is "computationally feasible" for small $p$.

In another paper [16], we will give many examples where the good bound obtained by Theorem 1.4 is computationally feasible as well as discuss in detail a connection to lifting supersingular elliptic curves to CM elliptic curves coming from the Deuring lift [6] and a correspondence of Gross [10]. We will list here the choices of $p$ for which the bound is computationally feasible for every $\theta \in M_{3 / 2}^{+}(4 p)$, namely $p=11, p=17$, and $p=19$. For simplicity, when we write $D$ henceforth, $-D$ will be a fundamental discriminant and $d$ will denote that $-d$ is any discriminant.

Theorem 1.5. The following table lists all eligible integers $T$ (or the size of the set and the largest element) divisible at most once by $p$ not represented by the given quadratic form $Q:=[a, b, c, d, e, f]=a x^{2}+b y^{2}+c z^{2}+d x y+e x z+f y z$. Moreover, $d$ is represented if and only if $d p^{2}$ is represented and the supersingular elliptic curve corresponding to $Q$ lifts to an elliptic curve with $C M$ by $\boldsymbol{O}_{-D}$ if and only if $D$ is not in the set $T$.

\begin{tabular}{|l|l|l|}
\hline$p$ & Quadratic Form $Q$ & $T$ \\
\hline 11 & {$[4,11,12,0,4,0]$} & $\{3,67,235,427\}$ \\
& {$[3,15,15,-2,2,14]$} & $\# T=21, \max _{t \in T} t=11803$ \\
\hline 17 & {$[7,11,20,-6,4,8]$} & $\{3,187,643\}$ \\
& {$[3,23,23,-2,2,22]$} & $\# T=88, \max _{t \in T} t=89563$ \\
\hline 19 & {$[7,11,23,-2,6,10]$} & $\{4,19,163,760,1051\}$ \\
& {$[4,19,20,0,4,0]$} & $\# T=40, \max _{t \in T} t=27955$. \\
\hline
\end{tabular}


In Section 4, we deal with $-d$ not fundamental, using the Shimura lift [27], the Hecke operators (cf. [23]), and Deligne's optimal bound for integral weight cusp forms [5]. Fixing a discriminant $-d$ and exploring the representability of $d^{\prime}=d F^{2}$, the Hecke operators lead to an equivalence between the following system of equations and the representability of $d$ by $Q$.

Theorem 1.6. There is a recursively defined polynomial $P_{k, m, \pm 1}(x)$ and a function $Q^{\prime}(x, y)$, defined as a rational function in terms of the Fourier coefficients of the decomposition of $\theta$ and its Shimura lift, such that $a_{\theta}\left(d F^{2}\right)=0$ if and only if for every $f=\prod_{l} l^{r_{l}}$ dividing $F$ and $\tilde{f}=\prod_{l} l^{s_{l}}$ with $s_{l} \leq \frac{1}{2} v_{l}(d)$,

$$
\prod_{l \text { prime }} P_{r_{l}, s_{l},\left(\frac{-D}{l}\right)}(l)=Q^{\prime}(f, \tilde{f}) .
$$

Remark 1.7. The power of Theorem 1.6 is that the left side is growing like l, while the right side grows like $2 \sqrt{l}$, so that the resulting system of equations is seldom consistent.

Theorem 1.8. Fix a discriminant $-d$. Consider $\theta=E+\sum_{i} b_{i} g_{i}$, where $g_{i}$ are fixed Hecke eigenforms. If $a_{\theta}\left(d F^{2}\right)=0$ with $(F, p)=1$,

$$
F \ll_{\epsilon}(p-1)^{2+\epsilon}\left(\sum_{i=1}^{m}\left|b_{i}\right|\right)^{2+\epsilon} d^{\alpha+\epsilon},
$$

where $\alpha=\frac{6}{7}$ unconditionally, $\alpha=-\frac{1}{7}$ under GRH for Dirichlet L-functions, and $\alpha=-\frac{1}{2}$ under GRH for Dirichlet L-functions and L-functions for weight 2 newforms.

Combining Theorem 1.1 and Theorem 1.8 along with an argument of Duke [8] to remove the dependence on $\theta$ yields the following result.

Theorem 1.9. Let $p$ be a prime, $\theta \in M_{3 / 2}^{+}(4 p)$, and $\epsilon>0$. Assuming GRH for Dirichlet $L$-functions and weight 2 modular forms, $a_{\theta}(d) \neq 0$ for every discriminant $-d$ with $\left(\frac{-d}{p}\right) \neq 1$ and $p^{2} \nmid d$ such that

$$
d \gg_{\epsilon} p^{14+\epsilon} \text {. }
$$

Here the implied constant depends only on $\epsilon$ and is effective. Moreover, $a_{\theta}(d)=0$ if and only if $a_{\theta}\left(d p^{2}\right)=0$.

We now interpret Theorem 1.9 in terms of lifts of supersingular elliptic curves.

Theorem 1.10. Let $p$ be a prime and $\epsilon>0$. Assume GRH for Dirichlet L-functions and weight 2 modular forms. Let $E / \overline{\mathbb{F}_{p}}$ be a supersingular elliptic curve. Then $E$ lifts to a elliptic curve over a number field with $C M$ by $\boldsymbol{O}_{-D}$ for every $\left(\frac{-D}{p}\right) \neq 1$ with

$$
D \gg_{\epsilon} p^{14+\epsilon} \text {. }
$$

A more detailed introduction and further details of the proofs may be found in [15]. 


\section{ACKNOWLEDGEMENTS}

This research was obtained while the author was a student at the University of Wisconsin - Madison. The author would like to thank T.H. Yang for his help and guidance. We would also like to thank K. Bringmann, D. Farmer, K. Ono, and J. Rouse for useful comments. The author thanks the reviewer for a very extensive and helpful report.

\section{Notation and Brief Overview of the Proof of Theorem 1.4}

We give useful notation here as well as the background needed for Theorem 1.4. We will denote half integral weight cusp forms with lower case letters and their Shimura lift with capital letters.

Let $p$ be an odd prime and $\theta \in M_{3 / 2}^{+}(4 p)$ be a theta function. Suppose $-D<-4$ is

a fundamental discriminant with $\left(\frac{-D}{p}\right) \neq 1$ and $a_{\theta}(D)=0$. Using an explicit formula for the coefficients of the Eisenstein series (cf. [10]) in terms of the class number, and Dirichlet's Class Number Formula [4],

$$
a_{E}(D)=\frac{12}{(p-1) 2^{v_{p}(D)}} H(-D)=\frac{12}{(p-1)} \cdot \frac{L(1) \cdot \sqrt{D}}{\pi 2^{v_{p}(D)}} .
$$

Here $L(s)$ is the Dirichlet $L$-function of the character $\chi(n):=\chi_{-D}(n)=\left(\frac{-D}{n}\right)$ and $v_{p}(D)$ is the power of $p$ dividing $D$. Using equation (1.1) we have decomposed the cuspidal part into $\sum_{i=1}^{m} b_{i} g_{i}$, where $g_{i}$ are a fixed set of Hecke eigenforms. Plugging in and using Schwarz's inequality yields

$$
\frac{12}{(p-1) \pi 2^{v_{p}(D)}} \cdot|L(1)| \cdot \sqrt{D} \leq \sqrt{\sum_{i=1}^{m}\left|b_{i}\right|^{2}} \sqrt{\sum_{i=1}^{m}\left|a_{g_{i}}(D)\right|^{2}} .
$$

A variant of the Kohnen-Zagier formula (3.1) gives $\left|a_{g_{i}}(D)\right|^{2}=c_{i} 2^{-v_{p}(D)} D^{\frac{1}{2}} \cdot L_{i}(1)$, where

$$
c_{i}:=\frac{\left|a_{g_{i}}\left(m_{i}\right)\right|^{2}}{L\left(G_{i}, m_{i}, 1\right) m_{i}^{\frac{1}{2}}}
$$

with $m_{i}$ the first coefficient of $g_{i}$ such that $a_{g_{i}}\left(m_{i}\right) \neq 0$ with $\left(p, m_{i}\right)=1$, and

$$
L_{i}(s):=L\left(G_{i},-D, s\right):=\sum_{n=1}^{\infty} \frac{\chi(n) a_{G_{i}}(D)}{n^{s}} .
$$

is the $L$ series of $G_{i}$ twisted by the character $\chi$, where $G_{i}$ is the normalized Shimura lift of $g_{i}$ (the unique newform with the same eigenvalues as $g_{i}$ normalized so that $a_{G_{i}}(1)=1$. 
This gives

$$
\frac{12}{(p-1) \pi 2^{\frac{v_{p}(D)}{2}}} \cdot D^{\frac{1}{4}} \leq \sqrt{\sum_{i=1}^{m}\left|b_{i}\right|^{2}} \sqrt{\sum_{i=1}^{m} c_{i} \frac{L_{i}(1)}{L(1)^{2}}} .
$$

As in [24], we define

$$
F(s):=F_{i}(s):=\left(\frac{\sqrt{q}}{2 \pi}\right)^{s-1} \frac{L_{i}(s) \Gamma(s)}{L(s) L(2-s)},
$$

where $q$ is the conductor of $L_{i}$. Notice that $F(1)=\frac{L_{i}(1)}{L(1)^{2}}$. By the functional equation of $L_{i}(s)$ (assuming without loss of generality that the sign of the functional equation is +1 as we automatically get a better bound if $\left.L_{i}(1)=0\right)$, we know that $F(s)=F(2-s)$ and GRH for Dirichlet $L$-functions implies that $F(s)$ is analytic for $\frac{1}{2}<\operatorname{Re}(s)<\frac{3}{2}$. Therefore, for $\frac{1}{2}<\operatorname{Re}(s)=\sigma<\frac{3}{2}$ fixed, we know by the Phragmen-Lindelöf principle that the maximum is attained on the boundary of $\operatorname{Re}(s)=\sigma$ and $\operatorname{Re}(s)=2-\sigma$. Thus, for $1<\sigma<\frac{3}{2}$, the functional equation gives

$$
F(1) \leq \max _{t}|F(\sigma+i t)|
$$

We will bound $L(s)$ from below in Section 6 and $L_{i}(s)$ from above in Section 7 .

For $d \in \mathbb{N}$, with prime factorization $d=\prod_{l} l^{e_{l}}$, we will denote for notational ease

$$
\Omega(d):=\sum_{l} e_{l}, v_{l}(d)=e_{l}, v(d)=\#\left\{l: e_{l}>0\right\}, \text { and } \sigma_{k}(d)=\sum_{n \mid d} n^{k} .
$$

We recall the Euler constant

$$
\gamma:=-\frac{\Gamma^{\prime}}{\Gamma}(1) \approx .5772
$$

and denote the Riemann Zeta function by $\zeta(s)$. Finally, we denote

$$
\psi(x):=\sum_{n \leq x} \Lambda(n) .
$$

\section{A Kohnen-Zagier Type Formula}

Let $N$ be odd and square-free and let $g \in S_{k+1 / 2}^{\text {new }}(4 N)$ be a newform in Kohnen's plus space. Let $G \in S_{2 k}^{n e w}(N)$ be the Shimura lift of $g$ normalized so that $a_{G}(1)=1$. Let $w_{l}$ be the sign of the Atkin-Lehner involution $W_{l}$ for each prime $l$ dividing $N$.

Lemma 3.1. Let $(-1)^{k} D$ be a fundamental discriminant such that for each prime divisor $l$ of $N$, either $\left(\frac{D}{l}\right)=w_{l}$ or $\left(\frac{D}{l}\right)=0$. Then

$$
\frac{\left|a_{g}(D)\right|^{2}}{<g, g>}=2^{v\left(\frac{N}{(N, D)}\right)} \cdot \frac{(k-1) !}{\pi^{k}} \cdot D^{k-1 / 2} \cdot \frac{L\left(G,(-1)^{k} D, k\right)}{<G, G>},
$$


Remark 3.2. If the conditions of Lemma 3.1 are not satisfied, then Kohnen proved in [18] that $a_{g}(D)=0$.

Proof. This follows Kohnen's proof in [17], where the result is shown whenever $(D, l)=1$. Noting that $\left(\frac{(-1)^{k} D}{t}\right)=0$ for $t \mid \operatorname{gcd}(D, N)$, before evaluating $\left(G \mid W_{t}\right)(z)$ allows us to remove the relatively prime condition. Further details may be found in [15].

\section{Bounding Non-Fundamental Discriminant Coefficients}

In this section we employ the power of the Hecke operators and the Shimura lift to obtain information about $-d$ non-fundamental. The argument also repeatedly uses the simple fact that $a_{\theta}\left(D l^{2}\right)=0$ implies $a_{\theta}(D)=0$. Notice that many of the results in this section do not require GRH.

Lemma 4.1. Fix a fundamental discriminant $-D$ and $F$ with $(F, p)=1$. Define $F^{\prime}:=$ $\prod_{l \mid F} l$. Then the coefficients of the newform $g_{i} \in S_{\frac{3}{2}}^{+}(4 p)$ satisfy

$$
\left|a_{g_{i}}\left(D F^{2}\right)\right| \leq \sigma_{-\frac{1}{2}}\left(F^{\prime}\right) F^{\frac{1}{2}} \sigma_{0}(F)\left|a_{g_{i}}(D)\right|,
$$

where $\sigma_{k}(n)$ is defined in equation (2.7).

Proof. First note that if $a_{g_{i}}(D)=0$, then $a_{g_{i}}\left(D F^{2}\right)=0$ by the Hecke operators, so the result follows trivially.

We will use here the $D$-th Shimura correspondence [27] instead of the Shimura lift, similar to the argument in [9]. We will show that if $R=l^{m}$ such that $m \geq 1$ and $(R, F)=1$, then

$$
a_{g_{i}}\left(D(F R)^{2}\right)=\frac{a_{g_{i}}\left(D F^{2}\right)}{a_{g_{i}}(D)}\left[a_{G_{D, i}}(R)-\left(\frac{-D}{l}\right) a_{G_{D, i}}\left(\frac{R}{l}\right)\right] .
$$

Using equation (4.2), we get the result easily by multiplicativity and Deligne's optimal bound [5] for integer weight eigenforms, which shows that

$$
\left|a_{G_{D, i}}(R)-\left(\frac{-D}{l}\right) a_{G_{D, i}}\left(\frac{R}{l}\right)\right| \leq\left(1+\frac{1}{l^{\frac{1}{2}}}\right) \sigma_{0}(R) R^{\frac{1}{2}}\left|a_{G_{D, i}}(1)\right| .
$$

We then use the fact that $a_{G_{D, i}}(1)=a_{g_{i}}(D)$. We now return to showing equation (4.2). Using the multiplicativity of the coefficients of $G_{D, i}$ normalized and the $D$-th Shimura correspondence, we obtain

$$
\begin{aligned}
& a_{G_{D, i}}(F) a_{G_{D, i}}(R)= a_{G_{D, i}}(F R) a_{G_{D, i}}(1)=\sum_{n \mid F R} a_{g_{i}}(D) a_{g_{i}}\left(D n^{2}\right)\left(\frac{-D}{F R / n}\right) \\
&=\left(\frac{-D}{l}\right) a_{G_{D, i}}(F) a_{G_{D, i}}(R / l)+\sum_{f \mid F} a_{g_{i}}(D) a_{g_{i}}\left(D R^{2} f^{2}\right)\left(\frac{-D}{F / f}\right) .
\end{aligned}
$$


Rearranging and using the $D$-th Shimura correspondence again for $a_{G_{D, i}}(F)$, we obtain

$$
0=\sum_{f \mid F}\left(a_{g_{i}}\left(D R^{2} f^{2}\right) a_{g_{i}}(D)-a_{g_{i}}\left(D f^{2}\right)\left[a_{G_{D, i}}(R)-\left(\frac{-D}{l}\right) a_{G_{D, i}}(R / l)\right]\right)\left(\frac{-D}{F / f}\right) .
$$

Hence equation (4.2) follows by induction on the number of divisors of $F$.

Theorem 4.2. If $a_{\theta}\left(D F^{2}\right)=0$ with $(F, p)=1$, then

$$
\frac{F^{\frac{1}{2}}}{2^{v(F)} \sigma_{-\frac{1}{2}}\left(F^{\prime}\right) \sigma_{0}(F)} \leq \frac{(p-1) \pi 2^{\frac{v_{p}(D)}{2}}}{12} D^{-\frac{1}{4}} \cdot\left(\sum_{i=1}^{m}\left|b_{i}\right|^{2}\right)^{\frac{1}{2}} \cdot\left(\sum_{i=1}^{m} c_{i} \frac{L_{i}(1)}{L(1)^{2}}\right)^{\frac{1}{2}} .
$$

Here $c_{i}$ and $b_{i}$ are given by Equations (2.3) and (1.1), respectively.

Proof. If $d=D F^{2}$ is not represented by $Q$, then

$$
a_{E}(d)=-\sum_{i=1}^{m} b_{i} a_{g_{i}}(d)
$$

We then use the index formula (see [3], Theorem 7.24, p. 146) and Schwarz's inequality to bound

$$
\frac{F}{2^{v(F)}} a_{E}(D) \leq \frac{a_{E}(d)}{a_{E}(D)} \cdot a_{E}(D) \leq \sqrt{\sum_{i=1}^{m}\left|b_{i}\right|^{2}} \sqrt{\sum_{i=1}^{m}\left|a_{g_{i}}(d)\right|^{2}} .
$$

We then plug in (4.1) to bound the right hand side in terms of $a_{g_{i}}(D)$ and then relate $a_{E}(D)$ with $L(1)$ by Dirichlet's Class Number Formula and $a_{g_{i}}(D)$ with $L_{i}(1)$ by the Kohnen-Zagier variant (3.1) as in equation (2.5).

Proof of Theorem 1.8(Assuming Theorem 1.4). The first part of Theorem 1.8 now follows directly by taking Duke's bound [7] for the cusp form to bound $a_{g_{i}}(D) \ll D^{\frac{3}{7}+\epsilon}$ and hence to bound $L_{i}(1)$ and using the trivial lower bound for $L(1)$. The additional assumptions give the corresponding (effective) optimal bounds for $L(1)$ and $L_{i}(1)$.

Theorem 1.6 involves showing a connection between $a_{\theta}\left(d f^{2}\right)=0$ and the following two recursively defined functions.

Definition 4.3. Set $m, n \in \mathbb{Z}$, and $\epsilon \in\{-1,0,1\}$. Define the polynomial $P_{n, m, \epsilon}(x)$ recursively as follows:

$$
P_{n, m, \epsilon}(x):= \begin{cases}0 & \text { if } n<0 \text { or } m<0, \\ 1 & \text { if } n=0, \\ (x-\epsilon) P_{n-1,1, \epsilon}(x)+\epsilon P_{n-1,0, \epsilon} & \text { if } m=0, n>0, \\ x P_{n-1,2, \epsilon}(x)+\left(\frac{x}{x-\epsilon}\right) P_{n-1,0, \epsilon} & \text { if } m=1, n>0, \\ x P_{n-1, m+1, \epsilon}(x)+P_{n-1, m-1, \epsilon} & \text { if } m \geq 1, n>0 .\end{cases}
$$


Definition 4.4. For a discriminant $-d$ with $\left(\frac{-d}{p}\right) \neq 1$ and $l$ a prime with $l^{2} \nmid d$, define

$$
Q_{n, m}(l):=\frac{\sum_{i} b_{i} a_{G_{i}}(l)^{n} a_{g_{i}}\left(d l^{2 m}\right)}{-a_{E}\left(d l^{2 m}\right)} .
$$

Theorem 4.5. Let $-d$ be a discriminant with associated fundamental discriminant $-D$ and $l \neq p$ prime. Then $a_{\theta}\left(d l^{2 m+2 n}\right)=0$ if and only if

$$
P_{r, s,\left(\frac{-D}{l}\right)}(l)=Q_{r, s}(l)
$$

for every $r \leq n$ and $s \leq m$.

Proof. When $n=0$, the result is clear. We proceed by induction on $n$. We note first that $a_{\theta}\left(d l^{2 m} l^{2 n+2}\right)=0$ if and only if $a_{\theta}\left(d l^{2 m+2} l^{2 n}\right)=0$. Therefore, by the inductive hypothesis, $a_{\theta}\left(d l^{2 m} l^{2 n+2}\right)=0$ if and only if

$$
P_{r, s,\left(\frac{-D}{l}\right)}(l)=Q_{r, s}(l),
$$

for every $r \leq n$ and $s \leq m+1$. These conditions match up with the assumptions above except when $s=m+1$. Thus, it suffices to show assuming $P_{r, s,\left(\frac{-D}{l}\right)}(l)=Q_{r, s}(l)$ for every $r \leq n$ and $s \leq m$ implies that

$$
P_{r, m+1,\left(\frac{-D}{l}\right)}(l)=Q_{r, m+1}(l),
$$

for every $r \leq n$, is equivalent to

$$
P_{n+1, s,\left(\frac{-D}{l}\right)}(l)=Q_{n+1, s}(l),
$$

for every $s \leq m$.

Let $r \leq n$ be given. Using the definition of $Q_{r, m+1}(l)$, we have

$$
Q_{r, m+1}(l)=\frac{\sum_{i} b_{i} a_{G_{i}}(l)^{r} a_{g_{i}}\left(D l^{2 m+2}\right)}{-a_{E}\left(D l^{2 m+2}\right)} .
$$

Since $g_{i}$ is a hecke Eigenform with $G_{i}$ the normalized Shimura lift, and $a_{G_{i}}(1)=1$, we have

$$
\begin{aligned}
& \frac{\sum_{i} b_{i} a_{G_{i}}(l)^{r} a_{g_{i}}\left(D l^{2 m+2}\right)}{-a_{E}\left(D l^{2 m+2}\right)}=\frac{\sum_{i} b_{i} a_{G_{i}}(l)^{r+1} a_{g_{i}}\left(D l^{2 m}\right)}{-a_{E}\left(D l^{2 m+2}\right)} \\
&-\left(\frac{-D l^{2 m}}{l}\right) \frac{\sum_{i} b_{i} a_{G_{i}}(l)^{r} a_{g_{i}}\left(D l^{2 m}\right)}{-a_{E}\left(D l^{2 m+2}\right)}-l\left(\frac{\sum_{i} b_{i} a_{G_{i}}(l)^{r} a_{g_{i}}\left(D l^{2 m-2}\right)}{-a_{E}\left(D l^{2 m+2}\right)}\right) .
\end{aligned}
$$


Now, we note by the index formula (see [3]) that

$$
\frac{-a_{E}\left(D l^{2 m+2}\right)}{-a_{E}\left(D l^{2 m}\right)}=l-\left(\frac{-D l^{2 m}}{l}\right) \text {. }
$$

Therefore, it follows that

$$
\begin{gathered}
\left(l-\left(\frac{-D l^{2 m}}{l}\right)\right) Q_{r, m+1}(l)=\frac{\sum_{i} b_{i} a_{G_{i}}(l)^{r+1} a_{g_{i}}\left(D l^{2 m}\right)}{-a_{E}\left(D l^{2 m}\right)} \\
-\left(\frac{-D l^{2 m}}{l}\right) \frac{\sum_{i} b_{i} a_{G_{i}}(l)^{r} a_{g_{i}}\left(D l^{2 m}\right)}{-a_{E}\left(D l^{2 m}\right)}-\frac{l}{l-\left(\frac{-D l^{2 m-2}}{l}\right)} \cdot \frac{\sum_{i} b_{i} a_{G_{i}}(l)^{r} a_{g_{i}}\left(D l^{2 m-2}\right)}{-a_{E}\left(D l^{2 m-2}\right)} \\
=Q_{r+1, m}(l)-\left(\frac{-D l^{2 m}}{l}\right) Q_{r, m}(l)-\frac{l}{l-\left(\frac{-D l^{2 m-2}}{l}\right)} \cdot Q_{r, m-1}(l) .
\end{gathered}
$$

Now, assume that $Q_{r, m+1}(l)=P_{r, m+1, \epsilon}$. By assumption, we also have $Q_{r, m}=P_{r, m, \epsilon}$ and $Q_{r, m-1}=P_{r, m-1, \epsilon}$. Therefore, rearranging the above formula gives

$Q_{r+1, m}(l)=\left(l-\left(\frac{-D l^{2 m}}{l}\right)\right) P_{r, m+1, \epsilon}(l)+\left(\frac{-D l^{2 m}}{l}\right) P_{r, m, \epsilon}(l)+\frac{l}{l-\left(\frac{-D l^{2 m-2}}{l}\right)} \cdot P_{r, m-1, \epsilon}(l)$.

If $m \geq 2$, then the right hand side is

$$
l P_{r, m+1, \epsilon}(l)+P_{r, m-1, \epsilon}(l)=P_{r+1, m, \epsilon}(l),
$$

as desired. If $m=1$, the right hand side is

$$
l P_{r, m+1, \epsilon}(l)+\left(\frac{-D}{l}\right) P_{r, m-1, \epsilon}(l)=P_{r+1, m, \epsilon}(l) .
$$

Notice that we used $l^{2} \nmid D$ above so that $\left(\frac{-D}{l}\right)=\left(\frac{-D^{\prime}}{l}\right)$. Finally, if $m=0$, we use the same observation above to see that the right hand side is

$$
\left(l-\left(\frac{D}{l}\right)\right) P_{r, m+1, \epsilon}(l)+\left(\frac{-D}{l}\right) P_{r, m, \epsilon}(l)=P_{r+1, m, \epsilon}(l) .
$$

The reverse direction follows by reversing the argument.

Theorem 4.6 (Theorem 1.6). Let $-d$ be a discriminant and $(F, p)=1$. Then $a_{\theta}\left(d F^{2}\right)=$ 0 if and only if for every $f$ dividing $F$, with $f=\prod_{l \text { prime }} l^{n_{l, f}}$ and $m_{l}:=\left\lfloor\frac{v_{l}(d)}{2}\right\rfloor$, we have

$$
\prod_{l \text { prime }} P_{r_{l}, s_{l},\left(\frac{-D}{l}\right)}(l)=\frac{\sum_{i} b_{i} \prod_{l \text { prime }} a_{G_{i}}(l)^{r_{l}} a_{g_{i}}\left(\frac{d}{\prod_{l \text { prime }} l^{2 s_{l}}}\right)}{-a_{E}\left(\frac{d}{\prod_{l \text { prime }} l^{2 s_{l}}}\right)},
$$


for every $r_{l} \leq n_{l, f}$ and $s_{l} \leq m_{l}$, where $-D$ is the fundamental discriminant corresponding to the discriminant $-d$.

Proof. For $F$ a prime power, this is exactly Theorem 4.5. The proof follows by induction on the number of prime divisors of $F$, following the same template as in the proof of Theorem 4.5.

Corollary 4.7. If $\theta=E+g$ with $g$ an eigenform, then $a_{\theta}\left(D F^{2}\right) \neq 0$ for every $F \nmid 6$ with $F \neq p^{n}$.

Proof. For $l>3$, Theorem 4.5 implies $2 \sqrt{l} \geq a_{G}(l)=l$ and the cases $l=2$ and $l=3$ follow from inconsistencies between the polynomials for $F=l$ and $F=l^{2}$ obtained from Theorem 1.6.

Corollary 4.8. If $\theta=E+b_{1} g_{1}+b_{2} g_{2}$, then for $l \geq 5$ a prime and $-D$ a discriminant,

$$
a_{\theta}\left(D l^{4}\right) \neq 0 .
$$

Moreover, if $q$ is another prime with $(q, 6 p l)=1$, then

$$
a_{\theta}\left(D l^{2} q^{2}\right) \neq 0
$$

Proof. This follows easily from contradictions in the system of equations that are obtained from Theorem 1.6, using $-D$ a fundamental discriminant. Details of the calculations may be found in [15].

\section{Review of the Work of Ono and Soundararajan}

In this section, we review some results of Ono and Soundararajan [24] in preparation for bounding $L(s)$ and $L_{i}(s)$ in the next two sections. Recall $\chi:=\chi_{-D}, L(s):=L(s, \chi)$, $L_{i}(s):=L\left(G_{i},-D, s\right)$, and $F(s)=F_{i}(s)$.

5.1. Explicit Formulas. We will use the following 2 lemmas from [24] for explicit formulas of $\frac{L^{\prime}}{L}(s)$ and $\frac{L_{i}^{\prime}}{L_{i}}(s)$. These formulas are derived by studying an integral and shifting the line of integration, giving $\frac{L^{\prime}}{L}(s)$ or $\frac{L_{i}^{\prime}}{L_{i}}(s)$ as one of the residues.

Lemma 5.1 (Ono-Soundararajan $[24]$ ). Assume that $L(s) \neq 0$. Then

$$
-\frac{L^{\prime}}{L}(s)=\boldsymbol{G}_{1}(s, \boldsymbol{X})+E_{s i g}(s)-\frac{L^{\prime}}{L}(s-1) \boldsymbol{X}^{-1}-R(s),
$$

where

$$
E_{s i g}(s)=\sum_{\rho} \boldsymbol{X}^{\rho-s} \Gamma(\rho-s), \text { and } R(s)=\frac{1}{2 \pi i} \int_{-\operatorname{Re}(s)-1 / 2-i \infty}^{-R e(s)-1 / 2+i \infty}-\frac{L^{\prime}}{L}(s+w) \Gamma(w) \boldsymbol{X}^{w} d w
$$


and

$$
\boldsymbol{G}_{1}(s, \boldsymbol{X}):=\sum_{n=1}^{\infty} \frac{\Lambda(n) \chi(n)}{n^{s}} e^{-n / \boldsymbol{X}},
$$

with $\Lambda$ the Von-Mangoldt function. Here $\rho$ denotes a nontrivial zero of $L(s)$.

Lemma 5.2 (Ono-Soundararajan [24]). If $L_{i}(s) \neq 0$, then

$$
-\frac{L_{i}^{\prime}}{L_{i}}(s)=\boldsymbol{F}_{1}(s, \boldsymbol{X})+R_{s i g}(s)+R_{t r i}(s)+R_{\text {ins }}(s),
$$

where

$$
\boldsymbol{F}_{1}(s, \boldsymbol{X}):=\sum_{n=1}^{\infty} \frac{\lambda_{i}(n) \chi(n)}{n^{s}} e^{-n / \boldsymbol{X}}
$$

with $\lambda_{i}$ defined such that for $\operatorname{Re}(s)>3 / 2$

$$
\begin{gathered}
-\frac{L_{i}^{\prime}}{L_{i}}(s)=\sum_{n=1}^{\infty} \frac{\lambda_{i}(n) \chi(n)}{n^{s}} \\
R_{s i g}(s)=\sum_{\rho_{i}} \boldsymbol{X}^{\rho_{i}-s} \Gamma\left(\rho_{i}-s\right), \quad R_{t r i}(s)=\sum_{n=0}^{\infty} \boldsymbol{X}^{-n-s} \Gamma(-n-s),
\end{gathered}
$$

and

$$
R_{\text {ins }}(s)=\sum_{n=1}^{\infty} \frac{(-\boldsymbol{X})^{-n}}{n !} \cdot \frac{L_{i}^{\prime}}{L_{i}}(s-n) .
$$

Here $\rho_{i}$ are the nontrivial zeros of $L_{i}$.

We will fix $i$ and investigate $F(s):=F_{i}(s)$. Then

$$
\frac{F^{\prime}}{F}(s)=\log \left(\frac{\sqrt{q}}{2 \pi}\right)+\frac{L_{i}^{\prime}}{L_{i}}(s)+\frac{\Gamma^{\prime}}{\Gamma}(s)-\frac{L^{\prime}}{L}(s)+\frac{L^{\prime}}{L}(2-s) .
$$

5.2. Bounds for $\frac{\Gamma^{\prime}}{\Gamma}$. We will need bounds for $\frac{\Gamma^{\prime}}{\Gamma}$, and will use the bounds obtained in $[24]$.

Lemma 5.3 (Ono-Soundararajan [24]). Set $s=x+i y$.

1) If $x \geq 1$, then

$$
\left|\frac{\Gamma^{\prime}}{\Gamma}(s)\right| \leq \frac{11}{3}+\frac{\log \left(1+x^{2}\right)}{2}+\frac{\log \left(1+y^{2}\right)}{2} .
$$

2) If $x>0$, then we have the bound

$$
R e\left(\frac{\Gamma^{\prime}}{\Gamma}(s)\right) \leq \frac{\Gamma^{\prime}}{\Gamma}(x)+\frac{y^{2}}{x|s|^{2}}+\log \left(\frac{|s|}{x}\right) .
$$


3) In general, one has

$$
\left|\frac{\Gamma^{\prime}}{\Gamma}(s)\right| \leq \frac{9}{2}+\frac{1}{<x>(1-<x>)}+\log (2+|x|)+\frac{\log \left(1+y^{2}\right)}{2},
$$

where $\left\langle x>:=\min _{n \in \mathbb{N}}|x+n|\right.$.

Lemma 5.4. If $0<x<1$, then

$$
\left|\frac{\Gamma^{\prime}}{\Gamma}(s)\right| \leq \frac{11}{3}+\frac{\log (2)}{2}+\frac{1}{x}+\frac{\log \left(1+y^{2}\right)}{2} .
$$

Proof. This follows from

$$
\left|\frac{\Gamma^{\prime}}{\Gamma}(s)\right| \leq \frac{1}{|s|}+\left|\frac{\Gamma^{\prime}}{\Gamma}(s+1)\right|
$$

and Lemma 5.3, since $\frac{1}{|s|} \leq \frac{1}{x}$ and $\log \left(1+x^{2}\right) \leq \log (2)$.

Lemma 5.5 (Hadamard's Factorization Formula, Ono-Soundararajan [24]). If $L(s) \neq 0$ then

$$
R e\left(\frac{L^{\prime}}{L}(s)\right)=-\frac{1}{2} \log \left(\frac{m}{\pi}\right)-\frac{1}{2} \operatorname{Re}\left(\frac{\Gamma^{\prime}}{\Gamma}\left(\frac{s+1}{2}\right)\right)+\sum_{\rho} \operatorname{Re}\left(\frac{1}{s-\rho}\right),
$$

where the sum is taken over all non-trivial zeros $\rho$ of $L(s)$ and $m$ is the conductor of $\chi$.

Additionally, if $L_{i}(s) \neq 0$ then

$$
R e\left(\frac{L_{i}^{\prime}}{L_{i}}(s)\right)=-\frac{1}{2} \log \left(\frac{q}{4 \pi^{2}}\right)-R e\left(\frac{\Gamma^{\prime}}{\Gamma}(s)\right)+\sum_{\rho_{i}} R e\left(\frac{1}{s-\rho_{i}}\right),
$$

where the sum is taken over all non-trivial zeros $\rho_{i}$ of $L_{i}(s)$ and $q$ is the conductor of $L_{i}(s)$.

\section{Bounding $L(s)$ From Below}

Fix $1<\sigma<\frac{3}{2}$. For notational ease, define $s:=\sigma+i t, s_{0}:=2-\sigma+i t$, and $\sigma_{0}:=\operatorname{Re}\left(s_{0}\right)$. Fix $\boldsymbol{X}>e^{\gamma+\frac{1}{3}-\sigma}$, recalling the Euler constant $\gamma$ in (2.8). In preparation for bounding $F(s)$, in this section we will find a bound from below for $\log \left(\left|\frac{L\left(s_{0}\right)}{L(s)}\right|\right)$, depending on $\boldsymbol{X}$, $t$, and $\sigma$. A philosophical explanation of the techniques used and further details may be found in $[15]$.

Set

$$
\delta(\boldsymbol{X}):=\max _{y} \int_{\sigma_{0}-1 / 2}^{\sigma-1 / 2} \boldsymbol{X}^{-u} \Gamma(-u+i y) d u \mid \cdot\left(\frac{1}{2} \log \frac{y^{2}+(\sigma-1 / 2)^{2}}{y^{2}+\left(\sigma_{0}-1 / 2\right)^{2}}\right)^{-1}
$$


We note that since $\Gamma$ decays exponentially in $y$ and the other term only has polynomial growth, $\delta(\boldsymbol{X})$ is well defined. Recall our definition (5.1) of $\boldsymbol{G}_{1}$ and denote

$$
\boldsymbol{G}(s, \boldsymbol{X}):=\sum_{n=2}^{\infty} \frac{\Lambda(n) \chi(n)}{n^{s} \log (n)} e^{-n / \boldsymbol{X}}=-\int \boldsymbol{G}_{1}(w, \boldsymbol{X}) d w .
$$

The goal of this section is to prove the following.

Theorem 6.1. Assume GRH for Dirichlet L-functions. Let $\chi$ be a primitive Dirichlet character of conductor $m$ and let $L(s)=L(s, \chi)$. For $\boldsymbol{X}>e^{\gamma+\frac{1}{\frac{3}{2}-\sigma}}$ we have

$$
\begin{array}{r}
\log \frac{\left|L\left(s_{0}\right)\right|}{|L(s)|} \geq \frac{\boldsymbol{X}}{\boldsymbol{X}+1-\delta(\boldsymbol{X}) \boldsymbol{X}}\left(\operatorname{Re}\left(\boldsymbol{G}\left(s_{0}, \boldsymbol{X}\right)\right)-\operatorname{Re}(\boldsymbol{G}(s, \boldsymbol{X}))+c_{\theta, \sigma, \boldsymbol{X}, 1}\right. \\
\left.+c_{\theta, \sigma, \boldsymbol{X}, t, 1}+c_{\theta, \sigma, \boldsymbol{X}, m, 1}\right)
\end{array}
$$

where the functions $c_{\theta, \sigma, \boldsymbol{X}, 1}, c_{\theta, \sigma, \boldsymbol{X}, t, 1}$, and $c_{\theta, \sigma, \boldsymbol{X}, m, 1}$ are given by

$$
\begin{aligned}
c_{\theta, \sigma, \boldsymbol{X}, 1}: & =-\left(\sigma-\sigma_{0}\right) \frac{\left|\Gamma\left(3 / 2-\sigma_{0}\right)\right|}{2 \pi \boldsymbol{X}^{\sigma_{0}+1 / 2}}\left(\frac{\pi}{r} \frac{759}{100}+\frac{\pi \log (226)}{2 r^{2}}+\frac{2}{\sqrt{15} r^{2}}\right) \\
& -\frac{\sigma-\sigma_{0}}{2 \boldsymbol{X}}\left(\frac{22}{3}+\frac{2}{\sigma_{0}}\right)-\frac{1-\sigma_{0}}{2 \boldsymbol{X}}\left(\frac{\log \left(1+\left(\frac{3-\sigma_{0}}{2}\right)^{2}\right)}{2}+\frac{\log (2)}{2}+\frac{2}{\sigma_{0}}\right) \\
& -\left(\frac{\sigma-1}{2 \boldsymbol{X}}\right)\left(\log (2)+\frac{2}{3-\sigma_{0}}\right)-2 \delta(\boldsymbol{X}) \log \frac{\Gamma\left(\frac{\sigma+1}{2}\right)}{\Gamma\left(\frac{\sigma_{0}+1}{2}\right)}-2 \delta(\boldsymbol{X}) \log \left(\frac{\sigma+1}{\sigma_{0}+1}\right),
\end{aligned}
$$

with $r=\sqrt{\left(\sigma_{0}+1 / 2\right)\left(\sigma_{0}-1 / 2\right)}$,

$$
c_{\theta, \sigma, \boldsymbol{X}, t, 1}:=\left(\sigma-\sigma_{0}\right)\left(-\frac{\left|\Gamma\left(3 / 2-\sigma_{0}\right)\right|}{4 r \boldsymbol{X}^{\sigma_{0}+1 / 2}}-\frac{1}{2 \boldsymbol{X}}-\left(\frac{\delta(\boldsymbol{X})}{2}\right)\right) \log \left(1+t^{2}\right),
$$

and finally

$$
c_{\theta, \sigma, \boldsymbol{X}, m, 1}:=\left|\sigma-\sigma_{0}\right|\left(\frac{\boldsymbol{X}-1}{\boldsymbol{X}^{2}}-\frac{\delta(\boldsymbol{X})}{2}\right) \log \left(\frac{m}{\pi}\right) .
$$

Proof. Integrating from $s_{0}$ to $s$ in Lemma 5.1 yields

$\log \left(\frac{L\left(s_{0}\right)}{L(s)}\right)=\boldsymbol{G}\left(s_{0}, \boldsymbol{X}\right)-\boldsymbol{G}(s, \boldsymbol{X})+\int_{s_{0}}^{s} E_{s i g}(w) d w-\int_{s_{0}}^{s} R(w) d w+\frac{1}{\boldsymbol{X}} \log \left(\frac{L\left(s_{0}-1\right)}{L(s-1)}\right)$.

We will take the real part of both sides, and bound each term. 
(i) We will first bound $\int_{s_{0}}^{s} R(w) d w$. We will show

$$
\begin{gathered}
\operatorname{Re}\left(-\int_{s_{0}}^{s} R(w) d w\right) \geq-\left|\int_{s_{0}}^{s} R(w) d w\right| \geq-\left|\sigma-\sigma_{0}\right|\left[\frac{\log \left(\frac{m}{\pi}\right)}{\boldsymbol{X}^{2}}\right. \\
\left.\quad+\frac{\left|\Gamma\left(3 / 2-\sigma_{0}\right)\right|}{2 \pi \boldsymbol{X}^{\sigma_{0}+1 / 2}}\left(\frac{\pi}{r}\left(\frac{759}{100}+\frac{1}{2} \log \left(1+t^{2}\right)\right)+\frac{\pi \log (226)}{2 r^{2}}+\frac{2}{\sqrt{15} r^{2}}\right)\right] .
\end{gathered}
$$

Rewriting $w=u+i t$ and plugging in the functional equation for $\frac{L^{\prime}}{L}$ gives

$$
\begin{array}{r}
R(w)=\frac{1}{2 \pi i} \int_{-u-1 / 2-i \infty}^{-u-1 / 2+i \infty} \Gamma(z) \boldsymbol{X}^{z}\left[\log \left(\frac{m}{\pi}\right)+\frac{1}{2} \cdot \frac{\Gamma^{\prime}}{\Gamma}\left(\frac{2-z-w}{2}\right)\right. \\
\left.+\frac{1}{2} \cdot \frac{\Gamma^{\prime}}{\Gamma}\left(\frac{1+z+w}{2}\right)+\frac{L^{\prime}}{L}(1-z-w)\right] d z .
\end{array}
$$

Consider $z=-u-1 / 2+i y$. Since $\operatorname{Re}\left(\frac{1+z+w}{2}\right)=\frac{1}{4}, \operatorname{Re}\left(\frac{2-z-w}{2}\right)$ and $\frac{t+y}{2}=\operatorname{Im}\left(\frac{1+z+w}{2}\right)=$ $-\operatorname{Im}\left(\frac{2-z-w}{2}\right)$, we use Lemma 5.4 to bound the term with $\frac{1+z+w}{2}$ and Lemma 5.3 to bound the term with $\frac{2-z-w}{2}$. Since $\operatorname{Re}\left(\frac{1+z+w}{2}\right)=\frac{3}{2}$, we can bound the $\frac{L^{\prime}}{L}$ term by $\left|\frac{\zeta^{\prime}}{\zeta}\left(\frac{3}{2}\right)\right| \leq \frac{151}{100}$. Noting that

$$
\log \left(1+\frac{(t+y)^{2}}{4}\right) \leq \log \left(1+t^{2}+y^{2}+t^{2} y^{2}\right)=\log \left(1+t^{2}\right)+\log \left(1+y^{2}\right)
$$

we obtain

$$
\begin{array}{r}
\left|\frac{L^{\prime}}{L}(1-z-w)+\frac{1}{2} \frac{\Gamma^{\prime}}{\Gamma}\left(\frac{1+z+w}{2}\right)+\frac{1}{2} \frac{\Gamma^{\prime}}{\Gamma}\left(\frac{2-z-w}{2}\right)\right| \\
\leq \frac{151}{100}+\frac{11}{6}+\frac{\log (2)}{4}+2+\frac{11}{6}+\frac{\log (1+25 / 16)}{4}+\frac{1}{2} \log \left(1+t^{2}\right)+\frac{1}{2} \log \left(1+y^{2}\right) \\
\leq \frac{759}{100}+\frac{1}{2} \log \left(1+t^{2}\right)+\frac{1}{2} \log \left(1+y^{2}\right) .
\end{array}
$$

Since $|\Gamma(x+i y)| \leq|\Gamma(x)|$, the functional equation for $\Gamma$ yields

$$
\left|\boldsymbol{X}^{z} \Gamma(z)\right|=\boldsymbol{X}^{-u-1 / 2} \cdot \frac{|\Gamma(z+2)|}{|z(z+1)|} \leq \boldsymbol{X}^{-u-1 / 2} \cdot \frac{|\Gamma(3 / 2-u)|}{(1 / 2+u)(u-1 / 2)+y^{2}} .
$$

It is easy to see that for $\boldsymbol{X}>e^{\gamma+\frac{1}{\frac{3}{2}-\sigma}}$, this function of $u$ on the right hand side decreases in $[1 / 2, \sigma]$, so we get that the maximum for $u \in\left[\sigma_{0}, \sigma\right]$ is attained at $u=\sigma_{0}$.

This gives the bound

$$
\left|\boldsymbol{X}^{z} \Gamma(z)\right| \leq \boldsymbol{X}^{-\sigma_{0}-1 / 2} \cdot \frac{\left|\Gamma\left(3 / 2-\sigma_{0}\right)\right|}{\left(1 / 2+\sigma_{0}\right)\left(\sigma_{0}-1 / 2\right)+y^{2}} .
$$


Furthermore, shifting the line of integration in the remaining term to the far left, noting that $-2<-\sigma-1 / 2<-u-1 / 2<-\sigma_{0}-1 / 2<-1$, then for $\boldsymbol{X}>1$,

$$
\frac{1}{2 \pi i} \int_{-u-1 / 2-i \infty}^{-u-1 / 2+i \infty} \Gamma(z) \boldsymbol{X}^{z} \log \left(\frac{m}{\pi}\right)=\log \left(\frac{m}{\pi}\right) \sum_{n=2}^{\infty} \frac{(-\boldsymbol{X})^{-n}}{n !} \leq \frac{\log \left(\frac{m}{\pi}\right)}{\boldsymbol{X}^{2}}
$$

Recall $r=\sqrt{\left(\sigma_{0}+1 / 2\right)\left(\sigma_{0}-1 / 2\right)}$. Plugging in the bounds from equations (6.3), (6.4), and (6.5) give

$$
\begin{aligned}
|R(w)| & \leq \frac{\log \left(\frac{m}{\pi}\right)}{\boldsymbol{X}^{2}}+\frac{\left|\Gamma\left(3 / 2-\sigma_{0}\right)\right|}{2 \pi \boldsymbol{X}^{\sigma_{0}+1 / 2}} \int_{-\infty}^{\infty} \frac{1}{y^{2}+r^{2}}\left(\frac{759}{100}+\frac{1}{2} \log \left(1+t^{2}\right)+\frac{1}{2} \log \left(1+y^{2}\right)\right) d y \\
& =\frac{\log \left(\frac{m}{\pi}\right)}{\boldsymbol{X}^{2}}+\frac{\left|\Gamma\left(3 / 2-\sigma_{0}\right)\right|}{2 \pi \boldsymbol{X}^{\sigma_{0}+1 / 2}}\left(\frac{\pi}{r}\left(\frac{759}{100}+\frac{1}{2} \log \left(1+t^{2}\right)\right)+\int_{0}^{\infty} \frac{\log \left(1+y^{2}\right)}{y^{2}+r^{2}} d y\right) .
\end{aligned}
$$

Splitting the remaining integral into the range 0 to 15 and 15 to $\infty$ gives

$$
|R(w)| \leq \frac{\log \left(\frac{m}{\pi}\right)}{\boldsymbol{X}^{2}}+\frac{\left|\Gamma\left(3 / 2-\sigma_{0}\right)\right|}{2 \pi \boldsymbol{X}^{\sigma_{0}+1 / 2}}\left(\frac{\pi}{r}\left(\frac{759}{100}+\frac{1}{2} \log \left(1+t^{2}\right)\right)+\frac{\pi \log (226)}{2 r^{2}}+\frac{2}{\sqrt{15} r^{2}}\right) .
$$

Since this is independent of $w$, integrating from $w=s_{0}$ to $w=s$ gives equation (6.2).

(ii) We will next find a bound for $\frac{1}{\boldsymbol{X}} \log \left|\frac{L\left(s_{0}-1\right)}{L(s-1)}\right|$. We will show

$$
\begin{aligned}
\frac{1}{\boldsymbol{X}} \log \left|\frac{L\left(s_{0}-1\right)}{L(s-1)}\right| \geq \frac{1}{\boldsymbol{X}}\left[\log \frac{|L(s)|}{\left|L\left(s_{0}\right)\right|}+\left|\sigma-\sigma_{0}\right| \log \left(\frac{m}{\pi}\right)\right. \\
-\frac{1-\sigma_{0}}{2}\left(\frac{22}{3}+\frac{\log \left(1+\left(\frac{3-\sigma_{0}}{2}\right)^{2}\right)}{2}+\log \left(1+t^{2}\right)+\frac{\log (2)}{2}+\frac{2}{\sigma_{0}}\right) \\
\left.\quad-\frac{\sigma-1}{2}\left(\frac{22}{3}+\log \left(1+t^{2}\right)+\log (2)+\frac{2}{3-\sigma_{0}}+\frac{2}{\sigma_{0}}\right)\right]
\end{aligned}
$$

Again using the functional equation for $\frac{L^{\prime}}{L}$ and noting that $\left|L\left(2-s_{0}\right)\right|=|L(s)|$ and $|L(2-s)|=\left|L\left(s_{0}\right)\right|$, we have

$$
\log \frac{\left|L\left(s_{0}-1\right)\right|}{|L(s-1)|} \geq \log \frac{|L(s)|}{\left|L\left(s_{0}\right)\right|}+\left|\sigma-\sigma_{0}\right| \log \left(\frac{m}{\pi}\right)-\frac{1}{2} \int_{s_{0}}^{s}\left|\frac{\Gamma^{\prime}}{\Gamma}\left(\frac{3-w}{2}\right)+\frac{\Gamma^{\prime}}{\Gamma}\left(\frac{w}{2}\right)\right||d w| .
$$

For $w \in\left(\sigma_{0}, 1\right)$ we use equation $(5.3)$ to bound $\Gamma^{\prime} / \Gamma((3-w) / 2)$ while we use equation (5.6) to bound the other term and again to bound both terms when $w \in[1, \sigma]$, yielding equation (6.6). 
(iii) Finally, we bound $\int_{s_{0}}^{s} E_{\text {sig }}(w) d w$. We will show here

$$
\begin{aligned}
& \int_{s_{0}}^{s} \operatorname{Re}\left(E_{\mathrm{sig}}(w)\right) d w \geq-\delta(\boldsymbol{X}) \cdot\left[\log \frac{|L(s)|}{\left|L\left(s_{0}\right)\right|}+\frac{\sigma-\sigma_{0}}{2} \log \left(\frac{m}{\pi}\right)+2 \log \frac{\Gamma\left(\frac{\sigma+1}{2}\right)}{\Gamma\left(\frac{\sigma_{0}+1}{2}\right)}\right. \\
& \left.+2 \log \left(\frac{\sigma+1}{\sigma_{0}+1}\right) \cdot \frac{t^{2}}{t^{2}+\left(\sigma_{0}+1\right)^{2}}+\left(\frac{\sigma+1}{2}-\frac{\sigma_{0}+1}{2}\right) \log \left(1+\frac{t^{2}}{\left(\sigma_{0}+1\right)^{2}}\right)\right] .
\end{aligned}
$$

Since $\frac{1}{w-\rho}$ is analytic from $s_{0}$ to $s$, an individual zero $\rho:=1 / 2+i y$ contributes

$$
\begin{aligned}
\int_{s_{0}}^{s} \operatorname{Re}\left(\boldsymbol{X}^{\rho-w} \Gamma(\rho-w)\right) d w \geq- & \left|\int_{\sigma_{0}}^{\sigma} \boldsymbol{X}^{1 / 2-u} \Gamma(1 / 2-u+i(y-t)) d u\right| . \\
& \left(\log \frac{|s-\rho|}{\left|s_{0}-\rho\right|}\right)^{-1} \cdot \int_{s_{0}}^{s} \operatorname{Re}\left(\frac{1}{w-\rho}\right) d w .
\end{aligned}
$$

We have added the additional term (6.9), which is 1 , so that we may use $\operatorname{Re}\left(\frac{1}{w-\rho}\right)$ later in Hadamard's factorization formula (5.5).

Since the integral and the log term merely make up one such term for $y$ fixed, we know that they are bounded above by $\delta(\boldsymbol{X})$. Therefore, summing the contributions of all zeros gives us

$$
\int_{s_{0}}^{s} \operatorname{Re}\left(E_{\mathrm{sig}}(w)\right) d w \geq-\delta(\boldsymbol{X}) \int_{s_{0}}^{s} \sum_{\rho} \operatorname{Re} \frac{1}{w-\rho} d w .
$$

Integrating Hadamard's factorization formula (5.5) yields

$$
\int_{s_{0}}^{s} \sum_{\rho} \operatorname{Re}\left(\frac{1}{w-\rho}\right) d w=\log \frac{|L(s)|}{\left|L\left(s_{0}\right)\right|}+\frac{\sigma-\sigma_{0}}{2} \log \left(\frac{m}{\pi}\right)+\int_{s_{0}}^{s} \operatorname{Re}\left(\frac{\Gamma^{\prime}}{\Gamma}\left(\frac{w+1}{2}\right)\right) d w .
$$

We now use equation (5.4) to bound the $\Gamma^{\prime} / \Gamma$ term. After noting that for $w=u+i t$, $4\left(\frac{u+1}{2}\right)^{2}+t^{2} \geq t^{2}+\left(\sigma_{0}+1\right)^{2}$ and integrating, we obtain equation (6.8).

Finally, rearranging equations (6.2), (6.6), and (6.8) and combining the terms involving $\log \frac{L\left(s_{0}\right)}{L(s)}$ yields Theorem 6.1.

\section{Bounding $L_{i}(1)$ From ABOve}

We use the same notation as in Section 6. We also define $\sigma_{1}:=3-\sigma$ and $s_{1}:=\sigma_{1}+i t$. In addition, we will fix $1<\sigma_{2}<2$ and consider $s_{2}:=\sigma_{2}+i t$. In practice we will 
choose $\sigma_{2}>\sigma$, but the theorem holds in general. We will find a bound from above for $\log \left(\left|L_{i}(D)\right|\right)$, depending on $\boldsymbol{X}, t$, and $\sigma$. Recall our definition (5.2) of $\boldsymbol{F}_{1}$ and denote

$$
\boldsymbol{F}(w, \boldsymbol{X}):=\sum_{n=1}^{\infty} \frac{\lambda_{i}(n) \chi(n)}{n^{w} \log (n)} e^{-n / \boldsymbol{X}}=-\int \boldsymbol{F}_{1}(w, \boldsymbol{X}) d w
$$

Theorem 7.1. Assume GRH for L-functions of weight 2 newforms, and set $L_{i}(s):=$ $L\left(G_{i}, \chi, s\right)$ with $\chi$ a primitive character such that $L_{i}$ has conductor $q$. Then,

$$
\begin{array}{r}
\log \left|L_{i}(s)\right| \leq \frac{\boldsymbol{X}}{\boldsymbol{X}+1} \boldsymbol{F}(s, \boldsymbol{X})-\frac{\boldsymbol{X}((1+2 \gamma(\boldsymbol{X})) \alpha(\boldsymbol{X})-\gamma(\boldsymbol{X}) \beta(\boldsymbol{X}))}{(\boldsymbol{X}+1)(1+\gamma(\boldsymbol{X}))} \boldsymbol{F}_{1}\left(s_{2}, \boldsymbol{X}\right) \\
+\frac{\boldsymbol{X}}{\boldsymbol{X}+1}\left(c_{\theta, \sigma, \boldsymbol{X}, 2}+c_{\theta, \sigma, \boldsymbol{X}, t, 2}+c_{\theta, \sigma, \boldsymbol{X}, q, 2}\right)
\end{array}
$$

where

$$
\begin{aligned}
& c_{\theta, \sigma, \boldsymbol{X}, 2}:=\sum_{n=2}^{N} \frac{\left|\lambda_{i}(n)\right|}{n^{\sigma_{1}} \log (n)}\left(1-e^{-n / \boldsymbol{X}}\right)+2 \log \left(\zeta\left(\sigma_{1}-1 / 2\right)\right)-2 \sum_{n=1}^{N} \frac{\Lambda(n)}{n^{\sigma_{1}-1 / 2} \log (n)} \\
& +\max \left\{|\Gamma(\sigma)|,\left|\Gamma\left(\sigma_{1}\right)\right|\right\} \cdot \frac{\boldsymbol{X}^{1-\sigma}}{(\boldsymbol{X}-1) \log (\boldsymbol{X})} \\
& +\left|\log \left(\zeta\left(4-\sigma_{1}-1 / 2\right)\right)-\log (\zeta(4-\sigma-1 / 2))\right|\left(\frac{1}{\boldsymbol{X}^{2}}+\frac{1}{3 \boldsymbol{X}^{2}(\boldsymbol{X}-1)}\right) \\
& +\frac{2}{\boldsymbol{X}} \log (\zeta(3-\sigma-1 / 2))+\frac{2\left(\sigma_{1}-\sigma\right)}{3 \boldsymbol{X}^{2}}\left(\frac{49}{6}+\log (20)\right)+\frac{2}{\boldsymbol{X}} \log \frac{\Gamma\left(\sigma_{1}+1\right)}{\Gamma(\sigma+1)} \\
& +\frac{2\left(\log \frac{\sigma_{1}-1}{\sigma-1}-\log \frac{2-\sigma_{1}}{2-\sigma}\right)}{3 \boldsymbol{X}^{2}}+\frac{(1+2 \gamma(\boldsymbol{X})) \alpha(\boldsymbol{X})-\gamma(\boldsymbol{X}) \beta(\boldsymbol{X})}{(1+\gamma(\boldsymbol{X}))}\left(\frac{\Gamma^{\prime}}{\Gamma}\left(\sigma_{2}\right)+\frac{1}{\sigma_{2}}\right) \\
& +\alpha(\boldsymbol{X}) \frac{\left|\Gamma\left(-\sigma_{2}\right)\right| \boldsymbol{X}^{1-\sigma_{2}}}{\boldsymbol{X}-1}+\alpha(\boldsymbol{X}) \frac{3 \boldsymbol{X}+2}{3 \boldsymbol{X}^{2}}\left(\frac{49}{6}+\log (20)+\frac{1}{\left(2-\sigma_{2}\right)\left(\sigma_{2}-1\right)}\right. \\
& \left.+2\left|\frac{\zeta^{\prime}}{\zeta}\left(3-1 / 2-\sigma_{2}\right)\right|\right) \\
& c_{\theta, \sigma, \boldsymbol{X}, t, 2}:=\log \left(1+t^{2}\right)\left(\frac{2\left(\sigma_{1}-\sigma\right)}{3 \boldsymbol{X}^{2}}+\frac{\left(\sigma_{1}-\sigma\right)}{\boldsymbol{X}}+\frac{(1+2 \gamma(\boldsymbol{X})) \alpha(\boldsymbol{X})-\gamma(\boldsymbol{X}) \beta(\boldsymbol{X})}{2(1+\gamma(\boldsymbol{X}))}\right. \\
& \left.+\frac{3 \boldsymbol{X}+2}{\boldsymbol{X}^{2}} \alpha(\boldsymbol{X})\right)
\end{aligned}
$$




$$
\begin{aligned}
& c_{\theta, \sigma, \boldsymbol{X}, q, 2}:=\log \left(\frac{q}{4 \pi^{2}}\right)\left(\frac{(1+2 \gamma(\boldsymbol{X})) \alpha(\boldsymbol{X})-\gamma(\boldsymbol{X}) \beta(\boldsymbol{X})}{2(1+\gamma(\boldsymbol{X}))}\right. \\
& \left.+\frac{\gamma(\boldsymbol{X})(\alpha(\boldsymbol{X})-\beta(\boldsymbol{X}))}{(1+\gamma(\boldsymbol{X}))} \cdot \frac{\left|\Gamma\left(-\sigma_{2}\right)\right| \boldsymbol{X}^{1-\sigma_{2}}}{\boldsymbol{X}-1}+\frac{\alpha(\boldsymbol{X})}{\boldsymbol{X}}+\frac{\sigma_{1}-\sigma}{\boldsymbol{X}}\right) \\
& \gamma(\boldsymbol{X}):=\boldsymbol{X}^{1-\sigma_{2}} \cdot \max _{y}\left|\Gamma\left(1-\sigma_{2}+i y\right)\right|\left(\left(\sigma_{2}-1\right)+\frac{y^{2}}{\sigma_{2}-1}\right), \\
& \beta(\boldsymbol{X}):=\left\{\begin{array}{ll}
\frac{\left(\sigma_{2}-1\right) \boldsymbol{X}^{\sigma_{2}-1}}{\boldsymbol{X}^{\sigma_{2}-1}-\Gamma\left(2-\sigma_{2}\right)} \int_{\sigma}^{\sigma_{1}} \operatorname{Re}\left(\boldsymbol{X}^{1-u} \Gamma(1-u)\right) d u & \text { if } \boldsymbol{X}<\Gamma\left(2-\sigma_{2}\right)^{\frac{1}{\sigma_{2}-1}} \\
-\frac{\left(\sigma_{2}-1\right) \boldsymbol{X}^{\sigma_{2}-1}}{\boldsymbol{X}^{\sigma_{2}-1}+\Gamma\left(2-\sigma_{2}\right)} \int_{\sigma}^{\sigma_{1}} \operatorname{Re}\left(\boldsymbol{X}^{1-u} \Gamma(1-u)\right) d u & \text { if } \Gamma\left(2-\sigma_{2}\right)^{\frac{1}{\sigma_{2}-1}} \leq \boldsymbol{X}
\end{array},\right.
\end{aligned}
$$

and finally

$$
\begin{aligned}
\alpha(\boldsymbol{X}):= & \max _{y}\left|\int_{\sigma}^{\sigma_{1}}\left(\boldsymbol{X}^{1-u} \Gamma(1-u+i y)\right) d u-\left(\beta(\boldsymbol{X}) \boldsymbol{X}^{1-\sigma_{2}} \Gamma\left(1-\sigma_{2}+i y\right)\right)\right| \\
& \cdot\left(\left(\sigma_{2}-1\right)+\frac{y^{2}}{\sigma_{2}-1}\right) .
\end{aligned}
$$

Proof. Integrating both sides of Lemma 5.2 from $s$ to $s_{1}$ yields

$$
\log L_{i}(s)=\log L_{i}\left(s_{1}\right)+\boldsymbol{F}(s, \boldsymbol{X})-\boldsymbol{F}\left(s_{1}, \boldsymbol{X}\right)+\int_{s}^{s_{1}}\left(R_{\mathrm{sig}}(w)+R_{\mathrm{ins}}(w)+R_{\mathrm{tri}}(w)\right) d w .
$$

We take real parts of both sides to bound $\log \left|L_{i}(s)\right|$. Since $\left|\lambda_{i}(n)\right| \leq 2 \sqrt{n} \Lambda(n)$, we bound

$$
\log \left|L_{i}\left(s_{1}\right)\right|-\operatorname{Re}\left(\boldsymbol{F}_{1}\left(s_{1}, \boldsymbol{X}\right)\right) \leq\left(1-e^{-n / \boldsymbol{X}}\right) \sum_{n=2}^{N} \frac{\left|\lambda_{i}(n)\right|}{n^{\sigma_{1}} \log (n)}+\sum_{n=N+1}^{\infty} \frac{2 \Lambda(n)}{n^{\sigma_{1}-1 / 2} \log (n)}
$$

Notice that taking the logarithmic derivative of $\zeta$ and integrating yields

$$
\sum_{n=N+1}^{\infty} \frac{2 \Lambda(n)}{n^{\sigma_{1}-1 / 2} \log (n)}=2 \log \left(\zeta\left(\sigma_{1}-1 / 2\right)\right)-2 \sum_{n=1}^{N} \frac{\Lambda(n)}{n^{\sigma_{1}-1 / 2} \log (n)},
$$

which can easily be computed numerically with a computer.

(i) We first bound the contribution from the trivial zeros:

Since $1<\sigma<w<\sigma_{1}<2$ and $|\Gamma(-n-w)|<|\Gamma(-w)|$, the maximum is attained either at $s$ or $s_{1}$. Thus, we can factor out $\max \left\{|\Gamma(\sigma)|,\left|\Gamma\left(\sigma_{1}\right)\right|\right\}$ and integrate to obtain

$$
\int_{s}^{s_{1}} R_{\mathrm{tri}}(w) d w \leq \max \left\{|\Gamma(\sigma)|,\left|\Gamma\left(\sigma_{1}\right)\right|\right\} \cdot \frac{\boldsymbol{X}^{1-\sigma}}{(\boldsymbol{X}-1) \log (\boldsymbol{X})} .
$$


(ii) We now bound the contribution from the poles of $\Gamma$ : We will show

$$
\begin{gathered}
\int_{s}^{s_{1}} R_{\mathrm{ins}}(w) d w \leq\left|\log \left(\zeta\left(4-\sigma_{1}-1 / 2\right)\right)-\log (\zeta(4-\sigma-1 / 2))\right|\left(\frac{1}{\boldsymbol{X}^{2}}+\frac{1}{3 \boldsymbol{X}^{2}(\boldsymbol{X}-1)}\right) \\
-\frac{\log \left(\left|L_{i}(s)\right|\right)}{\boldsymbol{X}}+\frac{2}{\boldsymbol{X}} \log (\zeta(3-\sigma-1 / 2))+\frac{2\left(\sigma_{1}-\sigma\right)}{3 \boldsymbol{X}^{2}}\left(\frac{49}{6}+\log \left(1+t^{2}\right)+\log (20)\right) \\
+\frac{2\left(\log \frac{\sigma_{1}-1}{\sigma-1}-\log \frac{2-\sigma_{1}}{2-\sigma}\right)}{3 \boldsymbol{X}^{2}}+\frac{2}{\boldsymbol{X}} \log \frac{\Gamma\left(\sigma_{1}+1\right)}{\Gamma(\sigma+1)}+\frac{\sigma_{1}-\sigma}{\boldsymbol{X}} \log \left(1+t^{2}\right)+\frac{\left(\sigma_{1}-\sigma\right)}{\boldsymbol{X}} \log \frac{q}{4 \pi^{2}} .
\end{gathered}
$$

We use the functional equation to obtain

$$
\begin{array}{r}
\int_{s}^{s_{1}} R_{\text {ins }}(w) d w=\sum_{n=1}^{\infty}-\frac{(-\boldsymbol{X})^{-n}}{n !}\left[\log \frac{L_{i}\left(2+n-s_{1}\right)}{L_{i}(2+n-s)}+\left(\sigma-\sigma_{1}\right) \log \frac{q}{4 \pi^{2}}\right. \\
\left.+\int_{s}^{s_{1}}\left(\frac{\Gamma^{\prime}}{\Gamma}(2-w+n)+\frac{\Gamma^{\prime}}{\Gamma}(w-n)\right) d w\right] .
\end{array}
$$

First we note that

$$
\sum_{n=1}^{\infty} \frac{(-\boldsymbol{X})^{-n}}{n !}\left(\sigma-\sigma_{1}\right) \log \frac{q}{4 \pi^{2}} \leq \frac{\left(\sigma_{1}-\sigma\right) \log \frac{q}{4 \pi^{2}}}{\boldsymbol{X}} .
$$

Expanding $\log \frac{L_{i}\left(2+n-s_{1}\right)}{L_{i}(2+n-s)}$ and noting that for $n \geq 2, \frac{1}{m^{2+n-\sigma_{1}}}-\frac{1}{m^{2+n-\sigma}} \leq \frac{1}{m^{4-\sigma_{1}}}-\frac{1}{m^{4-\sigma}}$, we get the bound

$$
\begin{aligned}
& \sum_{n=2}^{\infty} \frac{(-\boldsymbol{X})^{-n}}{n !} \log \left|\frac{L_{i}\left(2+n-s_{1}\right)}{L_{i}(2+n-s)}\right| \\
& \quad \leq 2\left|\log \left(\zeta\left(4-\sigma_{1}-1 / 2\right)\right)-\log (\zeta(4-\sigma-1 / 2))\right|\left(\frac{1}{2 \boldsymbol{X}^{2}}+\frac{1}{6 \boldsymbol{X}^{2}(\boldsymbol{X}-1)}\right)
\end{aligned}
$$

For $n=1$, taking the real part and noting that $\left|L_{i}\left(3-s_{1}\right)\right|=\left|L_{i}(s)\right|$, we have

$$
(-\boldsymbol{X})^{-1} \log \frac{\left|L_{i}\left(3-s_{1}\right)\right|}{\left|L_{i}(3-s)\right|} \leq-\frac{\log \left(\left|L_{i}(s)\right|\right)}{\boldsymbol{X}}+\frac{2}{\boldsymbol{X}} \log (\zeta(3-\sigma-1 / 2)) .
$$

For $n \geq 1$ we have $\operatorname{Re}(2-w+n) \geq 1$, so we may use equation (5.3) to bound the term with that parameter. For $n \geq 2$ we use equation (5.5) to bound the other term, and for $n=1$ we may use equation (5.6). 
It remains to bound

$$
\int_{s}^{s_{1}}\left(\frac{\Gamma^{\prime}}{\Gamma}(2-w+n)+\frac{\Gamma^{\prime}}{\Gamma}(w-n)\right) d w .
$$

Since $1<\sigma \leq w \leq \sigma_{1}<2$, we know that $2-w+n \geq 1$ for all $n \geq 1$, so that we can use equation (5.3) to bound that term. We will use equation (5.5) to bound the term with $w-n$ for $n \geq 2$. For $n=1$, we have $u-n \in(0,1)$, so that we can use equation (5.6). We note that for $u=\operatorname{Re}(w)>1$ we have $\langle u-n\rangle(1-<u-n>)=(2-u)(u-1)$. This yields, for $n \geq 2$,

$$
\begin{aligned}
\mid \frac{\Gamma^{\prime}}{\Gamma}(2-w+n)+ & \frac{\Gamma^{\prime}}{\Gamma}(w-n) \mid \\
& \leq \frac{49}{6}+\log ((3+n-u)(2+n-u))+\log \left(1+t^{2}\right)+\frac{1}{(2-u)(u-1)} .
\end{aligned}
$$

For $n=1$, we have $u-1>0$ and $3-u>0$, so that we can use equation (5.4) and the functional equation to obtain

$$
\begin{aligned}
& \operatorname{Re}\left(\frac{\Gamma^{\prime}}{\Gamma}(w-1)\right) \leq \operatorname{Re}\left(\frac{\Gamma^{\prime}}{\Gamma}(w)\right)-\operatorname{Re}\left(\frac{1}{w-1}\right) \leq \operatorname{Re}\left(\frac{\Gamma^{\prime}}{\Gamma}(w)\right) \\
\leq & \frac{\Gamma^{\prime}}{\Gamma}(u)+\frac{t^{2}}{u\left(t^{2}+u^{2}\right)}+\frac{1}{2} \log \left(1+t^{2}\right) \leq \frac{\Gamma^{\prime}}{\Gamma}(u)+\frac{1}{u}+\frac{1}{2} \log \left(1+t^{2}\right)=\frac{\Gamma^{\prime}}{\Gamma}(u+1)+\frac{1}{2} \log \left(1+t^{2}\right)
\end{aligned}
$$

and

$$
\begin{aligned}
\operatorname{Re}\left(\frac{\Gamma^{\prime}}{\Gamma}(3-w)\right) \leq & \frac{\Gamma^{\prime}}{\Gamma}(3-u)+\frac{t^{2}}{(3-u)\left(t^{2}+(3-u)^{2}\right)}+\frac{1}{2} \log \left(1+t^{2}\right) \\
& \leq \frac{\Gamma^{\prime}}{\Gamma}(3-u)+\frac{1}{3-u}+\frac{1}{2} \log \left(1+t^{2}\right)=\frac{\Gamma^{\prime}}{\Gamma}(4-u)+\frac{1}{2} \log \left(1+t^{2}\right) .
\end{aligned}
$$

Since $u>1, \log ((3+n-u)(2+n-u)) \leq \log ((n+1)(n+2))<\frac{n !}{6} \log (20)$ for $n \geq 3$ so for $\boldsymbol{X}>2$,

$$
\begin{aligned}
-\sum_{n=1}^{\infty} \frac{(-\boldsymbol{X})^{-n}}{n !} \int_{s}^{s_{1}} \operatorname{Re}\left(\frac{\Gamma^{\prime}}{\Gamma}(2-w+n)\right. & \left.+\frac{\Gamma^{\prime}}{\Gamma}(w-n)\right) d w \\
\leq \frac{2\left(\sigma_{1}-\sigma\right)}{3 \boldsymbol{X}^{2}}\left(\frac{49}{6}+\log \left(1+t^{2}\right)+\right. & \log (20))+\frac{2\left(\log \frac{\sigma_{1}-1}{\sigma-1}-\log \frac{2-\sigma_{1}}{2-\sigma}\right)}{3 \boldsymbol{X}^{2}} \\
& +\frac{2}{\boldsymbol{X}} \log \frac{\Gamma\left(\sigma_{1}+1\right)}{\Gamma(\sigma+1)}+\frac{\left(\sigma_{1}-\sigma\right)}{\boldsymbol{X}} \log \left(1+t^{2}\right) .
\end{aligned}
$$


(iii) Finally we bound the contribution from the significant zeros of $L_{i}$ : We will show

$$
\begin{gathered}
\int_{s}^{s_{1}} \operatorname{Re}\left(R_{\mathrm{sig}}(w)\right) d w \leq\left(\frac{(1+2 \gamma(\boldsymbol{X})) \alpha(\boldsymbol{X})-\gamma(\boldsymbol{X}) \beta(\boldsymbol{X})}{1+\gamma(\boldsymbol{X})}\right)\left(\frac{1}{2} \log \left(\frac{q}{4 \pi^{2}}\right)\right. \\
\left.+\frac{1}{2} \log \left(1+t^{2}\right)+\frac{\Gamma^{\prime}}{\Gamma}\left(\sigma_{2}\right)+\frac{1}{\sigma_{2}}-\boldsymbol{F}_{1}\left(s_{2}, \boldsymbol{X}\right)\right)+\alpha(\boldsymbol{X}) \frac{\left|\Gamma\left(-\sigma_{2}\right)\right| \boldsymbol{X}^{1-\sigma_{2}}}{\boldsymbol{X}-1} \\
+\frac{\gamma(\boldsymbol{X})(\alpha(\boldsymbol{X})-\beta(\boldsymbol{X}))}{1+\gamma(\boldsymbol{X})} \cdot \frac{\left|\Gamma\left(-\sigma_{2}\right)\right| \boldsymbol{X}^{1-\sigma_{2}}}{\boldsymbol{X}-1} \log \left(\frac{q}{4 \pi^{2}}\right)+\alpha(\boldsymbol{X}) \frac{3 \boldsymbol{X}+2}{\boldsymbol{X}^{2}}\left(\frac{49}{6}+\log (20)\right. \\
\left.+\frac{1}{\left(2-\sigma_{2}\right)\left(\sigma_{2}-1\right)}+\log \left(1+t^{2}\right)+2\left|\frac{\zeta^{\prime}}{\zeta}\left(3-1 / 2-\sigma_{2}\right)\right|\right)+\frac{\alpha(\boldsymbol{X})}{\boldsymbol{X}} \log \left(\frac{q}{4 \pi^{2}}\right) .
\end{gathered}
$$

Fix an individiual zero $\rho:=1+i y$.

$$
\begin{aligned}
\int_{s}^{s_{1}} \operatorname{Re}\left(\boldsymbol{X}^{\rho-w} \Gamma(\rho-w)\right) d w & =\operatorname{Re}\left(\beta(\boldsymbol{X}) \boldsymbol{X}^{\rho-s_{2}} \Gamma\left(\rho-s_{2}\right)\right) \\
& +\int_{s}^{s_{1}} \operatorname{Re}\left(\boldsymbol{X}^{\rho-w} \Gamma(\rho-w)\right) d w-\operatorname{Re}\left(\beta(\boldsymbol{X}) \boldsymbol{X}^{\rho-s_{2}} \Gamma\left(\rho-s_{2}\right)\right) \\
& \leq \operatorname{Re}\left(\beta(\boldsymbol{X}) \boldsymbol{X}^{\rho-s_{2}} \Gamma\left(\rho-s_{2}\right)\right)+\alpha(\boldsymbol{X}) \operatorname{Re}\left(\frac{1}{s_{2}-\rho}\right) .
\end{aligned}
$$

Now, summing over all non-trivial zeros of $L_{i}$ gives the bound

$$
\int_{s}^{s_{1}} \operatorname{Re}\left(R_{\mathrm{sig}}(w)\right) d w \leq \beta(\boldsymbol{X}) \operatorname{Re}\left(R_{\mathrm{sig}}\left(s_{2}\right)\right)+\alpha(\boldsymbol{X}) \sum_{\rho} \operatorname{Re}\left(\frac{1}{s_{2}-\rho}\right) .
$$

Rearranging Lemma 5.5 gives

$$
\sum_{\rho} \operatorname{Re}\left(\frac{1}{s_{2}-\rho}\right)=\operatorname{Re}\left(\frac{L_{i}^{\prime}}{L_{i}}\left(s_{2}\right)\right)+\frac{1}{2} \log \left(\frac{q}{4 \pi^{2}}\right)+\operatorname{Re}\left(\frac{\Gamma^{\prime}}{\Gamma}\left(s_{2}\right)\right)
$$

We again use the exact formula for $\frac{L_{i}^{\prime}}{L_{i}}$ from Lemma 5.2 to obtain

$$
\frac{L_{i}^{\prime}}{L_{i}}\left(s_{2}\right)=-\boldsymbol{F}_{1}\left(s_{2}, \boldsymbol{X}\right)-R_{\mathrm{sig}}\left(s_{2}\right)-R_{\mathrm{tri}}\left(s_{2}\right)-R_{\mathrm{ins}}\left(s_{2}\right) .
$$

We again need to bound each of these.

Clearly, taking the absolute value and noting that $\left|\Gamma\left(-n-s_{2}\right)\right| \leq\left|\Gamma\left(-\sigma_{2}\right)\right|$, we have

$$
\left|R_{\mathrm{tri}}\left(s_{2}\right)\right| \leq\left|\Gamma\left(-\sigma_{2}\right)\right| \sum_{n=0}^{\infty} \boldsymbol{X}^{-n-\sigma_{2}}=\frac{\left|\Gamma\left(-\sigma_{2}\right)\right| \boldsymbol{X}^{1-\sigma_{2}}}{\boldsymbol{X}-1} .
$$


We next bound $R_{\text {ins }}\left(s_{2}\right)$. We use the functional equation of $\frac{L_{i}^{\prime}}{L_{i}}$ to obtain $(7.15)$

$R_{\mathrm{ins}}\left(s_{2}\right)=\sum_{n=1}^{\infty}-\frac{(-\boldsymbol{X})^{-n}}{n !}\left(\frac{L_{i}^{\prime}}{L_{i}}\left(n+2-s_{2}\right)+\frac{\Gamma^{\prime}}{\Gamma}\left(s_{2}-n\right)+\frac{\Gamma^{\prime}}{\Gamma}\left(n+2-s_{2}\right)+\log \left(\frac{q}{4 \pi^{2}}\right)\right)$.

We note that $\frac{L_{i}^{\prime}}{L_{i}}(w)=-\sum_{m=1}^{\infty} \frac{\lambda_{i}(m) \chi(m)}{m^{w}}$ with $\left|\lambda_{i}(m)\right| \leq 2 \sqrt{m} \Lambda(m)$ and again use equations (5.5), (5.3), the fact that $\frac{6 \log ((n+1)(n+2))}{n ! \log (20)} \leq 1$ for every $n \geq 3$, and $\boldsymbol{X}>2$ to obtain

$$
\begin{aligned}
\left|R_{\text {ins }}\left(s_{2}\right)\right| \leq \frac{1}{\boldsymbol{X}} \log \left(\frac{q}{4 \pi^{2}}\right)+\frac{3 \boldsymbol{X}+2}{3 \boldsymbol{X}^{2}}\left(\frac{49}{6}+\log (20)\right. & +\frac{1}{\left(2-\sigma_{2}\right)\left(\sigma_{2}-1\right)} \\
& \left.+\log \left(1+t^{2}\right)+2\left|\frac{\zeta^{\prime}}{\zeta}\left(3-1 / 2-\sigma_{2}\right)\right|\right) .
\end{aligned}
$$

We then use equation (5.4) to bound $\operatorname{Re}\left(\frac{\Gamma^{\prime}}{\Gamma}\left(s_{2}\right)\right) \leq \frac{\Gamma^{\prime}}{\Gamma}\left(\sigma_{2}\right)+\frac{1}{\sigma_{2}}+\frac{1}{2} \log \left(1+t^{2}\right)$. Combining the terms involving $\operatorname{Re}\left(R_{\mathrm{sig}}\left(s_{2}\right)\right)$, it remains to bound

$$
|\beta(\boldsymbol{X})-\alpha(\boldsymbol{X})| \operatorname{Re}\left(R_{\text {sig }}\left(s_{2}\right)\right) .
$$

We bound $\operatorname{Re}\left(R_{\text {sig }}\left(s_{2}\right)\right)$ similarly to the way that we bound $R_{\text {sig }}$ above. Bounding by the absolute value, each non-trivial zero $\rho$ of $L_{i}$ contributes at most

$$
\left|\boldsymbol{X}^{\rho-s_{2}} \Gamma\left(\rho-s_{2}\right)\right| \leq \boldsymbol{X}^{1-\sigma_{2}}\left|\Gamma\left(1-\sigma_{2}+i(y-t)\right)\right|\left(\left(\sigma_{2}-1\right)+\frac{(t-y)^{2}}{\sigma_{2}-1}\right) \cdot \operatorname{Re}\left(\frac{1}{s_{2}-\rho}\right) .
$$

We then bound $\gamma(\boldsymbol{X})$ so that we have shown, using the functional equation for $\frac{L_{i}^{\prime}}{L_{i}}$ and the exact formula from Lemma 5.2,

$$
\begin{gathered}
\operatorname{Re}\left(R_{\text {sig }}\left(s_{2}\right)\right)=\operatorname{Re} \sum_{\rho}\left(\boldsymbol{X}^{\rho-s_{2}} \Gamma\left(\rho-s_{2}\right)\right) \leq \gamma(\boldsymbol{X}) \operatorname{Re}\left(\sum_{\rho} \frac{1}{s_{2}-\rho}\right) \\
=\gamma(\boldsymbol{X})\left(\frac{1}{2} \log \left(\frac{q}{4 \pi^{2}}\right)+\operatorname{Re}\left(\frac{\Gamma^{\prime}}{\Gamma}\left(s_{2}\right)\right)+\operatorname{Re}\left(\frac{L_{i}^{\prime}}{L_{i}}\left(s_{2}\right)\right)\right)=\gamma(\boldsymbol{X}) . \\
\left(\left(\frac{1}{2}+\frac{\left|\Gamma\left(-\sigma_{2}\right)\right| \boldsymbol{X}^{1-\sigma_{2}}}{\boldsymbol{X}-1}\right) \log \left(\frac{q}{4 \pi^{2}}\right)+\operatorname{Re}\left(\frac{\Gamma^{\prime}}{\Gamma}\left(s_{2}\right)\right)-\boldsymbol{F}_{1}\left(s_{2}, \boldsymbol{X}\right)-\operatorname{Re}\left(R_{\mathrm{sig}}\left(s_{2}\right)\right)\right) .
\end{gathered}
$$

We have already bounded $\operatorname{Re}\left(\frac{\Gamma^{\prime}}{\Gamma}\left(s_{2}\right)\right)$, so combining the $\operatorname{Re}\left(R_{\text {sig }}\left(s_{2}\right)\right)$ terms yields

$$
\begin{aligned}
\left|\operatorname{Re}\left(R_{\text {sig }}\left(s_{2}\right)\right)\right| \leq \frac{\gamma(\boldsymbol{X})}{1+\gamma(\boldsymbol{X})}\left(\left(\frac{1}{2}\right.\right. & \left.+\frac{\left|\Gamma\left(-\sigma_{2}\right)\right| \boldsymbol{X}^{1-\sigma_{2}}}{\boldsymbol{X}-1}\right) \log \left(\frac{q}{4 \pi^{2}}\right) \\
& \left.+\frac{\Gamma^{\prime}}{\Gamma}\left(\sigma_{2}\right)+\frac{1}{\sigma_{2}}+\frac{1}{2} \log \left(1+t^{2}\right)-\boldsymbol{F}_{1}\left(s_{2}, \boldsymbol{X}\right)\right) .
\end{aligned}
$$


The inequalities (7.14), (7.16), and (7.18) bound the terms in equation (7.13). Noting that $\alpha(\boldsymbol{X}) \geq \beta(\boldsymbol{X})$ because plugging $y=0$ into the term we are maximizing in $\alpha(\boldsymbol{X})$ gives exactly $\beta(\boldsymbol{X})$, since $\left(\sigma_{2}-1\right) \Gamma\left(1-\sigma_{2}\right)=-\Gamma(2-\sigma)$, we get equation (7.10).

8. Fundamental Discriminants and Bounds for Weight $3 / 2$ Cusp Forms

In this section we show how to find a bound $D_{\theta, \sigma, \sigma_{2}}$ such that for every fundamental discriminant $-D$ with $\left(\frac{-D}{p}\right) \neq 1$ and $D>D_{\theta, \sigma, \sigma_{2}}$ we have $a_{\theta}(D)>0$. Thus, combining this result with Section 4 gives the result for all discriminants.

8.1. Bounds for Fundamental Discriminants and Half Integer Weight Cusp Forms. We now proceed to show how bounds for $\alpha(\boldsymbol{X}), \gamma(\boldsymbol{X})$, and $\delta(\boldsymbol{X})$ are obtained.

Lemma 8.1. Fix a finite number of intervals $\left[y_{0, n}, y_{1, n}\right]$ with $0 \leq y_{0, n}<y_{1, n}<\infty$ such that $\bigcup_{n=1}^{m}\left[y_{0, n}, y_{1, n}\right] \cup\left[y_{1, m}, \infty\right)=[0, \infty)$. Then

$$
\begin{aligned}
\delta(\boldsymbol{X}) \leq \max & \left\{\max _{n \leq m} \int_{\sigma_{0}-1 / 2}^{\sigma-1 / 2} \boldsymbol{X}^{-u}\left|\Gamma\left(-u+i y_{0, n}\right)\right| d u \cdot\left(\frac{1}{2} \log \frac{y_{1, n}^{2}+(\sigma-1 / 2)^{2}}{y_{1, n}^{2}+\left(\sigma_{0}-1 / 2\right)^{2}}\right)^{-1},\right. \\
& \left.\int_{\sigma_{0}-1 / 2}^{\sigma-1 / 2} \boldsymbol{X}^{-u}\left|\Gamma\left(2-u+i y_{1, m}\right)\right| \frac{2}{\left(\left(\sigma_{0}-\frac{1}{2}\right)^{2}+y_{1, m}^{2}\right) \log \left(\frac{\left(\sigma-\frac{1}{2}\right)^{2}+y_{1, m}^{2}}{\left(\sigma_{0}-\frac{1}{2}\right)^{2}+y_{1, m}^{2}}\right)}\right\} .
\end{aligned}
$$

For $\gamma(\boldsymbol{X})$, we obtain the bound

$$
\begin{aligned}
\gamma(\boldsymbol{X}) \leq \boldsymbol{X}^{1-\sigma_{2}} \cdot \max \left\{\max _{n \leq m}\left|\Gamma\left(1-\sigma_{2}+i y_{0, n}\right)\right|\right. & \left(\left(\sigma_{2}-1\right)+\frac{y_{1, n}^{2}}{\sigma_{2}-1}\right) \\
& \left.\left|\Gamma\left(3-\sigma_{2}+i y_{1, m}\right)\right| \frac{1}{\sigma_{2}-1}\right\}
\end{aligned}
$$

Finally, for $\alpha(\boldsymbol{X})$ we obtain

$$
\begin{aligned}
\alpha(\boldsymbol{X}) \leq \max \left\{\max _{n \leq m} \int_{\sigma}^{\sigma_{1}} \boldsymbol{X}^{1-u}\left|\Gamma\left(1-u+i y_{0, n}\right)\right|\left(\left(\sigma_{2}-1\right)+\frac{y_{1, n}^{2}}{\sigma_{2}-1}\right)\right. \\
-\beta(\boldsymbol{X}) \boldsymbol{X}^{1-\sigma_{2}} \Gamma\left(1-\sigma_{2}+i y_{1, n}\right)\left(\left(\sigma_{2}-1\right)+\frac{y_{0, n}^{2}}{\sigma_{2}-1}\right), \\
\left.\qquad \int_{\sigma}^{\sigma_{1}} \boldsymbol{X}^{1-u}\left|\Gamma\left(3-u+i y_{1, m}\right)\right|\left(\frac{\left(\sigma_{2}-1\right)+\frac{y_{1, m}^{2}}{\sigma_{2}-1}}{(\sigma-1)^{2}+y_{1, m}^{2}}\right)\right\} .
\end{aligned}
$$


Remark 8.2. Taking the limit $\boldsymbol{X} \rightarrow \infty$ in the lemma with the intervals $[0,1]$ and $[1, \infty)$, we see that $\lim _{\boldsymbol{X} \rightarrow \infty} \delta(\boldsymbol{X})=0$ and $\lim _{\boldsymbol{X} \rightarrow \infty} \alpha(\boldsymbol{X})=0$ after noting that $\lim _{\boldsymbol{X} \rightarrow \infty} \beta(\boldsymbol{X})=0$.

Proof. We will show the result for $\delta(\boldsymbol{X})$, and the analogous calculation for $\alpha(\boldsymbol{X})$ and $\gamma(\boldsymbol{X})$ is left to the reader.

First define $\delta_{\left[y_{0}, y_{1}\right]}(\boldsymbol{X})$ to be the max taken in the interval $y_{0} \leq y \leq y_{1}$. Further, define $f(y):=\int_{\sigma_{0}-1 / 2}^{\sigma-1 / 2} \boldsymbol{X}^{-u}|\Gamma(-u+i y)| d u$ and $g(y):=\left(\frac{1}{2} \log \frac{y^{2}+(\sigma-1 / 2)^{2}}{y^{2}+\left(\sigma_{0}-1 / 2\right)^{2}}\right)^{-1}$. Notice first that $f$ is strictly decreasing in $y \geq 0$, which follows easily from the fact that $\Gamma(z)=\frac{e^{-\gamma z}}{z} \prod_{n=1}^{\infty}\left(1+\frac{z}{n}\right)^{-1} e^{\frac{z}{n}}$, while $g$ is strictly increasing. Therefore, noting that both functions are even in $y$, we fix $0 \leq y_{0}<y<y_{1}<\infty$, then pull the absolute value inside the integral to give

$$
\delta_{\left[y_{0}, y_{1}\right]}(\boldsymbol{X}) \leq f\left(y_{0}\right) g\left(y_{1}\right) .
$$

Now we deal with the case where $y_{1}=\infty$. The functional equation of $\Gamma(z)$ gives us

$$
f(y)=\int_{\sigma_{0}-1 / 2}^{\sigma-1 / 2} \boldsymbol{X}^{-u} \frac{|\Gamma(2-u+i y)|}{\left(u^{2}+y^{2}\right)^{1 / 2}\left((1-u)^{2}+y^{2}\right)^{1 / 2}} d u .
$$

We then bound $1-u \geq 1-\left(\sigma-\frac{1}{2}\right)=\frac{3}{2}-\sigma=\sigma_{0}-\frac{1}{2}$ and $u \geq \sigma_{0}-\frac{1}{2}$ and note that $|\Gamma(2-u+i y)|$ is decreasing in $y$ to get

$$
f(y) \leq\left(\int_{\sigma_{0}-1 / 2}^{\sigma-1 / 2} \boldsymbol{X}^{-u}\left|\Gamma\left(2-u+i y_{0}\right)\right| d u\right) \cdot \frac{1}{\left(\sigma_{0}-\frac{1}{2}\right)^{2}+y^{2}} .
$$

Defining $z:=\left(\sigma_{0}-\frac{1}{2}\right)^{2}+y^{2}$ and $a:=\left(\sigma-\frac{1}{2}\right)^{2}-\left(\sigma_{0}-\frac{1}{2}\right)^{2}$, we see that the remaining terms with $y$ form $\frac{2}{z \log \left(1+\frac{a}{z}\right)}$, which decreases in $z$ since $a>0$ and $z>0$.

We have now set up the framework to show our main theorems.

Proof of Theorem 1.1. We only need to show the result for a fundamental discriminant, since the results from Section 4 extend the bound for non-fundamental discriminants. We will first show the result for an orthonormal basis of newforms $g_{i}$. Consider the completion of $L_{i}(s)$, which we will normalize to

$$
\Lambda_{i}(s):=\left(\frac{q}{4 \pi^{2}}\right)^{\frac{s-1}{2}} \Gamma(s) L_{i}(s) .
$$

Again using the functional equation $\Lambda_{i}(s)= \pm \Lambda_{i}(2-s)$ and the analytic continuation, assuming without loss of generality that the sign of the functional equation is 1 , we can 
bound $L_{i}(1)=\Lambda_{i}(1) \leq \max _{t}\left|\Lambda_{i}(\sigma+i t)\right|$ with $1<\sigma<\frac{3}{2}$. We will choose $\sigma<1+\delta$. Since

$$
\lim _{\boldsymbol{X} \rightarrow \infty} \alpha(\boldsymbol{X})=0=\lim _{x \rightarrow \infty} \beta(\boldsymbol{X})
$$

This gives the power of $q$ from Theorem 7.1 as

$$
\begin{array}{r}
\frac{\boldsymbol{X}}{\boldsymbol{X}+1}\left[\frac{(1+2 \gamma(\boldsymbol{X})) \alpha(\boldsymbol{X})-\gamma(\boldsymbol{X}) \beta(\boldsymbol{X})}{2(1+\gamma(\boldsymbol{X}))}+\gamma(\boldsymbol{X}) \frac{\alpha(\boldsymbol{X})-\beta(\boldsymbol{X})}{1+\gamma(\boldsymbol{X})} \cdot \frac{\left|\Gamma\left(-\sigma_{2}\right)\right| \boldsymbol{X}^{1-\sigma_{2}}}{\boldsymbol{X}-1}\right. \\
\left.+\frac{\sigma_{1}-\sigma}{\boldsymbol{X}}+\frac{\alpha(\boldsymbol{X})}{\boldsymbol{X}}\right]+\frac{\sigma-1}{2}
\end{array}
$$

We then choose $\boldsymbol{X}_{0}$ large enough so that the resulting sum is less than $\delta$, which is possible because every term goes to zero except $\frac{\sigma-1}{2}<\frac{\delta}{2}$. Note that this choice of $\boldsymbol{X}_{0}$ only depends on $\sigma, \sigma_{2}$ and not on the level or the particular form. We then choose $\boldsymbol{X}_{1} \geq \boldsymbol{X}_{0}$ large enough so that the decay in $t$ from $\Gamma(s)$ overwhelms the polynomial growth from the $c_{\theta, \sigma, \boldsymbol{X}, t, 2}$ factor in Theorem 7.1. Choosing $N=1$ in $c_{\theta, \sigma, \boldsymbol{X}, 2}$, we now note that the resulting constant is independent of the form $L_{i}$, except for $\boldsymbol{F}(s, \boldsymbol{X})$ and $\boldsymbol{F}_{1}\left(s_{2}, \boldsymbol{X}\right)$. Bounding $\left|\lambda_{i}(n)\right| \leq 2 \sqrt{n} \Lambda(n)$ in each case, we are able to bound these independent of $L_{i}$ using Lemma 8.5 below. Since our choice of $\boldsymbol{X}_{1}$ did not depend on $L_{i}$, we obtain a bound from the Kohnen-Zagier variant (3.1) of

$$
\left|a_{g_{i}}(D)\right|^{2}=\frac{2^{-v_{p}(D)}}{\pi\left\|G_{i}\right\|^{2}} D^{\frac{1}{2}} L_{i}(1) \leq \frac{1}{\left\|G_{i}\right\|^{2}} \frac{2^{-v_{p}(D)}}{\pi} c_{\delta} N^{\delta} D^{\frac{1}{2}+2 \delta},
$$

where $c_{\delta}$ is the constant obtained from Theorem 7.1 after plugging in $\boldsymbol{X}_{1}$ and $\sigma$, multiplied by $|\Gamma(\sigma)|$, noting that $q \leq N D^{2}$. Thus, for the coefficient $\left|a_{g_{i}}(D)\right|$, the only dependence we have on $L_{i}$ is $\frac{N^{\frac{\delta}{2}}}{\left\|G_{i}\right\|}$, where $G_{i}$ is normalized to have $a_{G_{i}}(1)=1$.

We then take an arbitrary form $g$ with Petersson norm 1 and write $g=\sum_{i} b_{i} g_{i}$, where $g_{i}$ are an orthonormal basis, so that $\left\langle g_{i}, g_{j}\right\rangle=\delta_{i j}$ and $\sum_{i}\left|b_{i}\right|^{2}=1$ and each of $g_{i}$ satisfy the bound (8.4) from some level dividing the level $N$ of $g$. Then we simply take $c_{N}:=\max _{i} \frac{1}{\left\|G_{i}\right\|}$ and we get the desired result since there are at most $\operatorname{dim}\left(S_{2}\left(\Gamma_{0}(N)\right)\right)$ terms and $c_{N} \ll N^{\epsilon}$ from Hoffstein and Lockhart [12].

We use the following lemma of Duke [8] to prove Theorem 1.9.

Lemma 8.3 (Duke [8]). Fix $f \in S_{3 / 2}\left(\Gamma_{0}(N), \psi\right)$. Then

$$
\|f\|^{2} \ll \Gamma(\alpha) d(N) N^{2 \alpha} \sum_{n=1}^{\infty}\left|a_{f}(n)\right|^{2} n^{-\alpha},
$$

where $\alpha>\frac{1}{2}$ is any number so that the series exists, $d(\cdot)$ is the divisor function, and the constant is absolute. 
Proof of Theorem 1.9. Set $g:=\theta-E$. We will bound $E$ and $g$ independent of $\theta$. After noting that $\operatorname{dim}\left(S_{2}\left(\Gamma_{0}(p)\right)\right) \leq\left\lceil\frac{p+1}{12}\right\rceil \leq p$, Theorem 1.1 yields

$$
\left|a_{g}(d)\right| \ll_{\epsilon}\|g\| p^{\frac{1}{2}+\epsilon} d^{1 / 4+\epsilon} .
$$

Assuming GRH for Dirichlet $L$-functions, bounding the class number from below in equation (2.1) yields

$$
a_{E}(d) \gg_{\epsilon} \frac{1}{p} d^{1 / 2-\epsilon}
$$

It remains to use Lemma 8.3 to bound $\|g\|$ independent of $\theta$. Define

$$
M(G):=\sum_{Q^{\prime} \in G} \omega_{Q^{\prime}}^{-1},
$$

where $G$ is the genus containing $Q$ and $\omega_{Q^{\prime}}$ is the number of automorphs.

Since $a_{\theta_{Q^{\prime}}}(d) \geq 0$ for every $Q^{\prime}$, Siegel's averaging theorem (cf. [10]) gives

$$
\left|a_{g}(d)\right| \leq\left(M(G) \omega_{Q}+1\right) a_{E}(d) .
$$

Moreover, it is well known [21] that $\omega_{Q} \leq 48$, so $\left|a_{g}(d)\right| \ll M(G) a_{E}(d)$. The Eichler mass formula (cf. [10]) gives

$$
M(G)=\frac{p-1}{12} \leq p
$$

Therefore,

$$
\left|a_{g}(d)\right| \ll p a_{E}(d) \ll_{\epsilon} d^{\frac{1}{2}+\epsilon} .
$$

It is important to note here that the implied constant does not depend on $g$. The power of $d$ attained allows us to choose $\alpha=2+2 \epsilon$ in Lemma 8.3 for the convergence of the sum. Since we know that $N=p$ is the level, this yields

$$
\|g\|^{2} \ll_{\epsilon} p^{4+4 \epsilon} \text {. }
$$

Therefore,

$$
\left|a_{g}(d)\right| \ll_{\epsilon}\|g\| p^{\frac{1}{2}} d^{1 / 4+\epsilon} \ll_{\epsilon} p^{\frac{5}{2}+\epsilon} d^{1 / 4+\epsilon} .
$$

Combining equations (8.6) and (8.7), $a_{E}(d) \gg\left|a_{g}(d)\right|$ if $d \gg_{\epsilon} p^{14+\epsilon}$, as desired.

Theorem 8.4 (Theorem 1.4). Fix $\theta \in M_{3 / 2}^{+}(4 p)$. Assume GRH for Dirichlet L-series and $L$-functions of weight 2 newforms. For every $\boldsymbol{X}>e^{\gamma+\frac{1}{3 / 2-\sigma}}$ such that

$$
\begin{aligned}
\frac{\boldsymbol{X}}{\boldsymbol{X}+1}\left[\frac{(1+2 \gamma(\boldsymbol{X})) \alpha(\boldsymbol{X})-\gamma(\boldsymbol{X}) \beta(\boldsymbol{X})}{1+\gamma(\boldsymbol{X})}+\frac{2 \gamma(\boldsymbol{X})(\alpha(\boldsymbol{X})-\beta(\boldsymbol{X}))}{1+\gamma(\boldsymbol{X})} \cdot \frac{\left|\Gamma\left(-\sigma_{2}\right)\right| \boldsymbol{X}^{1-\sigma_{2}}}{\boldsymbol{X}-1}\right. \\
\left.+\frac{2\left(\sigma_{1}-\sigma\right)}{\boldsymbol{X}}+\frac{2 \alpha(\boldsymbol{X})}{\boldsymbol{X}}\right]+\frac{\boldsymbol{X}\left(\sigma-\sigma_{0}\right)}{\boldsymbol{X}+1-\delta(\boldsymbol{X}) \boldsymbol{X}}\left(\frac{\delta(\boldsymbol{X})}{2}-\frac{\boldsymbol{X}-1}{\boldsymbol{X}^{2}}\right)+(\sigma-1)<\frac{1}{2}
\end{aligned}
$$


there exists an effectively computable constant $D_{\sigma, \boldsymbol{X}}$ such that for all fundamental discriminants $-D<-D_{\sigma, \boldsymbol{X}}$ with $\left(\frac{-D}{p}\right) \neq 1$, one has $a_{\theta}(D) \neq 0$.

Moreover, such an $\boldsymbol{X}$ exists, so, assuming GRH for Dirichlet L-functions and weight 2 modular forms, there is an effectively computable constant $D_{\sigma}$ such that for all fundamental discriminants $-D<-D_{\sigma}$ with $\left(\frac{-D}{p}\right) \neq 1, a_{\theta}(D) \neq 0$.

Proof. By equation (2.5), it suffices to bound $F(s)$. By definition,

$$
\log |F(s)|=\log \left(\left|L_{i}(s)\right|\right)+\log (|\Gamma(s)|)-\log \left(\left|L\left(s_{0}\right)\right|\right)-\log (|L(s)|)+\frac{\sigma-1}{2} \log \frac{q}{4 \pi^{2}} .
$$

Plugging in our bounds from Theorems 6.1 and 7.1 yields

$$
\begin{gathered}
\log |F(s)| \leq \frac{\boldsymbol{X}}{\boldsymbol{X}+1} \boldsymbol{F}(s, \boldsymbol{X})-\frac{\boldsymbol{X}((1+2 \gamma(\boldsymbol{X})) \alpha(\boldsymbol{X})-\gamma(\boldsymbol{X}) \beta(\boldsymbol{X})}{(\boldsymbol{X}+1)(1+\gamma(\boldsymbol{X}))} \boldsymbol{F}_{1}\left(s_{2}, \boldsymbol{X}\right) \\
-\frac{\boldsymbol{X}}{\boldsymbol{X}-1-\delta(\boldsymbol{X}) \boldsymbol{X}}\left(\begin{array}{r}
\left.\operatorname{Re}\left(\boldsymbol{G}\left(s_{0}, \boldsymbol{X}\right)\right)-\operatorname{Re}(\boldsymbol{G}(s, \boldsymbol{X}))\right)+c_{\theta, \sigma, \boldsymbol{X}, 2}+c_{\theta, \sigma, \boldsymbol{X}, t, 2}+c_{\theta, \sigma, \boldsymbol{X}, q, 2} \\
-\left(c_{\theta, \sigma, \boldsymbol{X}, 1}+c_{\theta, \sigma, \boldsymbol{X}, t, 1}+c_{\theta, \sigma, \boldsymbol{X}, m, 1}\right)+\log |\Gamma(s)|-2 \log |L(s)| .
\end{array}\right.
\end{gathered}
$$

Using the fact that $q=p D^{2}$ and $m=D$, it remains to deal with $\log |\Gamma(s)|, 2 \log |L(s)|$, and the remaining terms involving $\boldsymbol{F}, \boldsymbol{F}_{1}$, and $\boldsymbol{G}$. We will combine the terms $c_{\theta, \sigma, \boldsymbol{X}, t, 1}$ and $c_{\theta, \sigma, \boldsymbol{X}, t, 2}$ and use the exponential decay of $\log |\Gamma(\sigma+i t)|$ to remove the dependence on $t$. Since $\sigma>1$, the term dealing with $\log |L(s)|$ may be bounded easily by

$$
\log |L(s)| \geq-\log |\zeta(\sigma)| .
$$

If we denote the sum of the terms involving $\boldsymbol{F}, \boldsymbol{F}_{1}$, and $\boldsymbol{G}$, using the notation used in [24], as

$$
\sum_{n=2}^{\infty} \operatorname{Re} \frac{\chi(n)}{n^{i t} \log (n)} v(n ; \boldsymbol{X})
$$

then, fixing a constant $N_{0}$, we may bound the first $N_{0}$ terms by a constant, and the remaining terms we will bound separately.

The contribution to $v(n ; \boldsymbol{X})$ from the terms involving $\boldsymbol{F}$ and $\boldsymbol{F}_{1}$ is

$$
e^{-n / x} \lambda_{i}(n) \chi(n) \cdot \frac{\boldsymbol{X}}{\boldsymbol{X}+1} \cdot\left(\frac{1}{n^{\sigma}}-a_{\boldsymbol{X}} \frac{\log (n)}{n^{\sigma_{2}}}\right),
$$

where $a_{\boldsymbol{X}}$ is the term in front of $\boldsymbol{F}_{1}$ above. Choosing $\sigma_{2}>\sigma$ and noting that $a_{\boldsymbol{X}}>0$, the asymptotic growth shows us that there exists an $N_{0}$ such that for $n>N_{0}$ we can bound the above term by

$$
\lambda_{i}(n) \chi(n) \cdot \frac{\boldsymbol{X}}{\boldsymbol{X}+1} \cdot\left(\frac{1}{n^{\sigma}}\right) .
$$


Now, using the fact that $\left|\lambda_{i}(n)\right| \leq 2 \Lambda(n) \sqrt{n}$, we have

$$
|v(n ; \boldsymbol{X})| \leq e^{-n / x}\left(\frac{2 \Lambda(n)}{n^{\sigma-1 / 2}}+b_{\boldsymbol{X}}\left(\frac{\Lambda(n)}{n^{\sigma_{0}}}-\frac{\Lambda(n)}{n^{\sigma}}\right)\right) \leq c_{\boldsymbol{X}} \frac{\Lambda(n)}{n^{\min \left(\sigma_{0}, \sigma-1 / 2\right)}} e^{-n / x}
$$

Therefore, since $c_{\boldsymbol{X}}$ is independent of $n$, it remains to bound sums of the form

$$
\boldsymbol{H}(\alpha, \boldsymbol{X}):=\sum_{n=N_{0}+1}^{\infty} \frac{\Lambda(n)}{n^{\alpha} \log (n)} e^{-n / x}
$$

We will need the following lemma which is a small generalization of a lemma from [24] to proceed with bounding the terms $n \rightarrow \infty$. Recall our definition $(2.9)$ of $\psi(x)$.

Lemma 8.5. Conditional upon the Riemann Hypothesis, one has for $0<\alpha<1$,

$$
\boldsymbol{H}(\alpha, \boldsymbol{X}) \leq \frac{e^{-N_{0} / \boldsymbol{X}}}{N_{0}^{\alpha} \log \left(N_{0}\right)}\left(c_{N_{0}} N_{0}-\psi\left(N_{0}\right)\right)+\frac{c_{N_{0}} \boldsymbol{X}^{1-\alpha}}{\log \left(N_{0}\right)} \Gamma\left(1-\alpha, N_{0} / \boldsymbol{X}\right)
$$

where

$$
\Gamma(x ; y):=\int_{y}^{\infty} t^{x-1} e^{-t} d t
$$

and $\psi(x)<c_{N_{0}} x$ for every $x \geq N_{0}$.

Proof. Since $\psi(x)$ jumps only at prime powers, it follows that

$$
\boldsymbol{H}(\alpha, \boldsymbol{X})=\int_{N_{0}}^{\infty} \frac{e^{-t / \boldsymbol{X}}}{t^{\alpha} \log (t)} d \psi(t)
$$

Using the results in Rosser and Schoenfeld [25], we have $\psi(x)<c_{N_{0}} x$ for $x \geq N_{0}-1 / 2$, and some $c_{N_{0}}>1$. Since Chebysheve showed that $\psi(x) \sim x$ is equivalent to the prime number theorem, shown by Hadamard and de la Vallée-Poussin (cf. [11]), this constant goes to 1 as $N_{0}$ goes to infinity, but Rosser and Schoenfeld give an explicit constant of

$$
1+\frac{\log \left(N_{0}\right)^{2}}{8 \pi \sqrt{N_{0}}}
$$

assuming the Riemann Hypothesis. 
Integration by parts now yields

$$
\begin{gathered}
\boldsymbol{H}(\alpha, \boldsymbol{X}) \leq \frac{e^{-N_{0} / \boldsymbol{X}}}{N_{0}^{\alpha} \log \left(N_{0}\right)}\left(c_{N_{0}} N_{0}-\psi\left(N_{0}\right)\right)+c_{N_{0}} \int_{N_{0}}^{\infty} \frac{e^{-t / \boldsymbol{X}}}{t^{\alpha} \log (t)} d t \\
\leq \frac{e^{-N_{0} / \boldsymbol{X}}}{N_{0}^{\alpha} \log \left(N_{0}\right)}\left(c_{N_{0}} N_{0}-\psi\left(N_{0}\right)\right)+\frac{c_{N_{0}}}{\log \left(N_{0}\right)} \int_{N_{0}}^{\infty} \frac{e^{-t / \boldsymbol{X}}}{t^{\alpha}} d t \\
=\frac{e^{-N_{0} / \boldsymbol{X}}}{N_{0}^{\alpha} \log \left(N_{0}\right)}\left(c_{N_{0}} N_{0}-\psi\left(N_{0}\right)\right)+\frac{c_{N_{0}} e^{-N_{0} / \boldsymbol{X}} \boldsymbol{X}^{1-\alpha}}{\log \left(N_{0}\right)} \Gamma\left(1-\alpha, N_{0} / \boldsymbol{X}\right) .
\end{gathered}
$$

We now return to the proof of Theorem 1.4. Notice that we have now shown that the only terms involving $D$ are the terms $\frac{\sigma-1}{2} \log \left(\frac{q}{4 \pi^{2}}\right), c_{\theta, \sigma, \boldsymbol{X}, q, 2}$ and $-c_{\theta, \sigma, \boldsymbol{X}, m, 1}$.

Investigating equation (2.5) shows that if the constant in front of $\log (D)$ is less than $\frac{1}{2}$, then we will have a result of the form $D \leq c$. Therefore, it only remains to show that there is an $\boldsymbol{X}$ such that the constant in front of $\log (D)$ is less than or equal to $\frac{1}{2}$. Plugging in $m=D$ and $q=p D^{2}$, and using our bounds for $\alpha(\boldsymbol{X}), \gamma(\boldsymbol{X})$ and $\delta(\boldsymbol{X})$ obtained in Lemma 8.1, we see that the limit of the power of $D$ as $\boldsymbol{X} \rightarrow \infty$ is $\sigma-1$. Since $\sigma<\frac{3}{2}$, such an $\boldsymbol{X}$ exists.

Remark 8.6. In practice, we will fix a constant $N_{0}$ and use cancellation between the first $N_{0}$ terms of the sum in equation (8.8) and the first $N_{0}$ terms of (8.9) to get a better explicit bound (see [16]).

\section{REFERENCES}

[1] M. Bhargava, On the Conway-Schneeberger fifteen theorem, Quadratic Forms and their applications (Dublin, 1999), 27-37, Contemp. Math 272, (Amer. Math. Soc., Providence, RI, 2000).

[2] M. Bhargava, J. Hanke, Universal quadratic forms and the 290-theorem, preprint.

[3] D. Cox, Primes of the form $x^{2}+n y^{2}$, Fermat, class field theory, and complex multiplication, (John Wiley \& Sons, Inc., New York, 1989).

[4] H. Davenport, Multiplicative Number Theory, (Springer-Verlag, New York, 1980).

[5] P. Deligne, La Conjecture de Weil I, Inst. Hautes Études Sci. Publ. Math. 43 (1974) 273-307.

[6] M. Deuring, Die Typen der Multiplikatorenringe elliptischer Funktionenkörper, Abh. Math. Sem., Hansischen Univ 14 (1941) 197-272.

[7] B. Duke, Hyperbolic distribution problems and half-integral weight Maass forms, Invent. Math. 92 (1988) 73-90.

[8] B. Duke, On ternary quadratic forms, J. Num. Theory 110(1) (2005) 37-43.

[9] B. Duke, R. Schulze-Pillot, Representation of integers by positive ternary quadratic forms and equidistribution of lattice points on ellipsoids., Invent. Math. 99 (1990) 49-57.

[10] B. Gross, Heights and the special values of $L$-series. Number theory (Montreal, Que., 1985), in CMS Conf. Proc., 7, (Amer. Math. Soc., Providence, RI, 1987), pp. 115-187.

[11] G. Hardy, E. Wright, Introduction to the Theory of Numbers, (The Clarendon Press, Oxford University Press, New York, 1979), pp. 340-342. 
[12] J. Hoffstein, P. Lockhart, Coefficients of Maass forms and the Siegel zero., Ann. of Math. 140 (1994), 161-181

[13] H. Iwaniec, Fourier coefficients of modular forms of half-integral weight, Invent. Math., 87 (1987) 385-401.

[14] H. Iwaniec, E. Kowalski, Analytic Number Theory. American Mathematical Society Colloquium Publications, 53. (American Mathematical Society, Providence, RI, 2004).

[15] B. Kane, PhD. Thesis, University of Wisconsin, May 2007.

[16] B. Kane, CM Liftings of Supersingular Elliptic Curves, preprint.

[17] W. Kohnen, Fourier coefficients of modular forms of half-integral weight. Math. Ann. 271(2) (1985) 237-268.

[18] W. Kohnen,Newforms of half-integral weight. J. Reine Angew. Math. 333 (1982) 32-72.

[19] T. Lam, The algebraic theory of quadratic forms. Mathematics Lecture Note Series, (W. A. Benjamin, Inc., Reading, Mass., 1973).

[20] J. Littlewood, On the Class Number of the corpus $P(\sqrt{-k})$, Proc. London Math. Soc. 27(2) (1928) 358-372.

[21] M. Newman, Integral matrices. Pure and Applied Mathematics, Vol. 45. (Academic Press, New York-London, 1972).

[22] J. Oesterlé, Nombres de classes des corps quadratiques imaginaires, Astérique 121-122 (1985) 309323.

[23] K. Ono, The web of modularity: arithmetic of the coefficients of modular forms and $q$-series. CBMS Regional Conference Series in Mathematics, 102. (Published for the Conference Board of the Mathematical Sciences, Washington, DC; by the American Mathematical Society, Providence, RI, 2004).

[24] K. Ono, K. Soundararajan, Ramanujan's ternary quadratic form, Invent. Math. 130 (3) (1997) $415-454$.

[25] J.B. Rosser, L. Schoenfeld, Sharper bounds for the Chebyshev functions $\theta(x)$ and $\psi(x)$. Collection of articles dedicated to Derrick Henry Lehmer on the occasion of his seventieth birthday. Math. Comp. 29 (1975) 243-269.

[26] R. Schulze-Pillot, Darstellung durch Spinorgeschlechter ternärer quadratischer Formen, J. Number Theory 12(4) (1980) 529-540.

[27] G. Shimura, On modular forms of half integral weight, Ann. of Math. 97(2) (1973) 440-481.

[28] C. L. Siegel, Über die Klassenzahl algebraischer Zahlkörper, Acta Arithmetica vol. 1 (1935) 83-86.

[29] W. Tartakowsky, Die Gesamtheit der Zahlen, die durch eine quadratische form $F\left(x_{1}, x_{2}, \ldots, x_{s}\right),(s \geq 4)$ darstellbar sind., Izv. Akad. Nauk SSSR 111-122 (1929), 165-196.

[30] J.-L. Waldspurger, Sur les coefficients de Fourier des formes modulaires de poids demi-entier, J. Math. Pures Appl. (9) 60(4) (1981) 375-484.

Department of Mathematics, Radboud Universiteit, Toernooiveld 1, 6525 Nijmegen, NETHERLANDS

E-mail address: bkane@science.ru.nl 